\title{
Optimisation methods for solving a large-scale avionics scheduling problem
}

\section{Emil Karlsson}

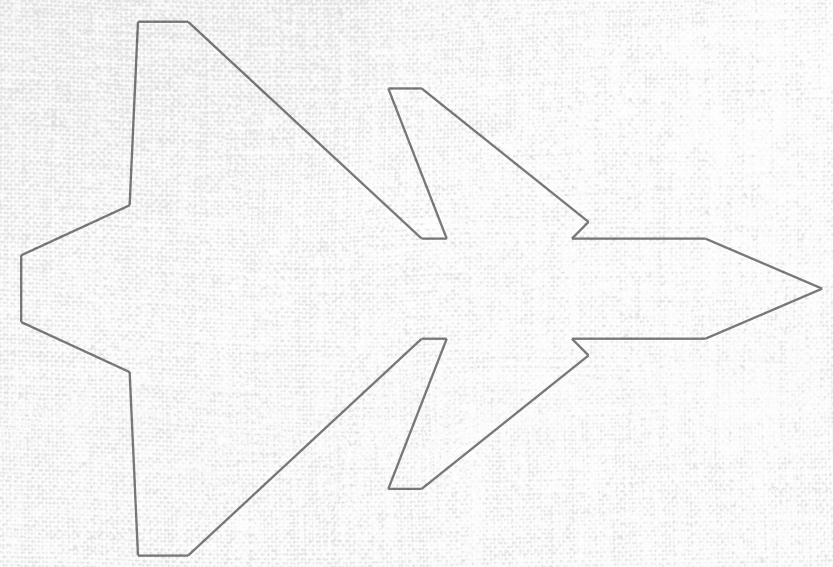



Linköping Studies in Science and Technology

Dissertations, No. 2136

\title{
Optimisation methods for solving a large-scale avionics scheduling problem
}

\author{
Emil Karlsson
}

1.UU UNNORDSG

Linköping University

Department of Mathematics

Division of Applied Mathematics

SE-581 83 Linköping, Sweden

Linköping 2021 
(cc) EY-NC This work is licensed under a Creative Commons AttributionNonCommercial 4.0 International License.

https://creativecommons.org/licenses/by-nc/4.0/

Edition 1:1

(C) Emil Karlsson, 2021

ISBN 978-91-7929-673-5

ISSN 0345-7524

URL http://urn.kb.se/resolve?urn=urn:nbn:se:liu:diva-173564

Published articles have been reprinted with permission from the respective copyright holder.

Typeset using $\mathrm{X}_{\mathrm{H}} \mathrm{T}_{\mathrm{E}} \mathrm{X}$

Printed by LiU-Tryck, Linköping 2021 


\begin{abstract}
Modern computer systems in aircraft are based on an integrated modular avionic architecture. In this architecture, software applications share hardware resources on a common avionic platform. Many functions in an aircraft are controlled by software and a failure in such software can have severe consequences. To avoid malfunction, there are many aspects to consider. One aspect is to ensure that the activities in the system get sufficient computing and network communication resources while being completed in time. This thesis contributes to addressing this challenge by studying an avionics scheduling problem proposed by Saab.
\end{abstract}

In this problem, tasks are performed on modules and the messages are sent on a communication network that links the modules. A schedule specifies start times for tasks on modules and the choices of time slots for messages on the communication network. For a schedule to be feasible, constraints must be respected: precedence constraints between tasks, timing constraints on tasks and messages, and communication network constraints involving both tasks and messages. For future platforms, it is expected that large-scale instances need to be solved. The methods introduced in this thesis solve instances with up to 55,000 tasks and 2500 messages.

The thesis includes three papers that introduce methods for solving the avionics scheduling problem and one paper that compares techniques to improve the performance of a specific type of decomposition method. The solution methods for the avionics problem differ in how the decomposition is made, how the subproblems are solved, and if the algorithm is guaranteed to find a feasible solution or not, given enough time. Together, the contributions of these papers give an insight into the structure of this type of avionics scheduling problem and how this structure can be exploited to construct efficient exact and matheuristic scheduling methods.

Paper A introduces a constraint generation procedure tailored for the avionics scheduling problem. In the constraint generation procedure, a preliminary, possibly infeasible, schedule is constructed by solving a master problem. This schedule is used to define a restriction of the problem, which is solved to find a complete schedule. If no schedule is found within a time-limit, constraints are added to the master problem, which is then solved again. This procedure relies on solving Mixed-Integer Programming (MIP) models. In Paper B, the constraint generation procedure is extended into a matheuristic, where the master problem is solved with a MIP-based adaptive large neighbourhood search. The methods in Paper A and Paper B can solve instances with up to 37,000 tasks and 1700 messages, and 55,000 tasks and 2500 messages, respectively.

Logic-Based Benders Decomposition (LBBD) algorithms are treated in Paper C and $\mathrm{Pa}-$ per D. In both papers, a MIP solver is applied to the master problem while a constraint programming solver is used for the subproblem. Paper $\mathrm{C}$ addresses the avionics scheduling problem and introduces a new acceleration technique for LBBD. This technique exploits information obtained during cut strengthening to make educated guesses about where feasible solutions might be found. With this technique, the LBBD algorithm outperforms the methods from Paper A and Paper B both with respect to solution time and RAM usage. Paper D investigates cut strengthening for LBBD in a wider context, by evaluating different cut-strengthening algorithms for LBBD on three problems from the literature. The computational evaluation shows that it is advantageous to invest time in finding strong cuts. 


\section{POPULÄRVETENSKAPLIG SAMMANFATTNING}

I dagens flygplan finns det stora mängder elektronik och funktioner som styrs av datorer, allt ifrån vad som visas på skärmarna för piloten till att flygplanet styrs av pilotens spakar. Detta gör att det är ytterst viktigt att de beräkningar som görs av datorerna inte bara är korrekta utan att de sker i rätt tid, annars kan det få allvarliga följder.

Under de senaste åren har flygplansindustrin gått från att använda federerade datorsystem, där varje dator ansvarar för en flygplansfunktion, till integrerade modulariserade datorsystem, där flera flygplansfunktioner implementeras på samma dator. Att implementera flera funktioner på samma dator gör det möjligt att minska mängden elektronik i flygplanet och därigenom nyttja elektroniken mer effektivt. En konsekvens är samtidigt att det blir mer utmanande att garantera att fel i en flygplansfunktion inte påverkar andra funktioner. Detta gör att det behövs andra strategier för att garantera att allt genomförs i rätt tid jämfört med om flygplansfunktionerna hade varit implementerade på varsin dator.

I fokus för denna avhandling är ett industriellt relevant schemaläggningsproblem av intresse för utveckling av framtida flygplanssystem, beskrivet av Saab. Som i en del att garantera att detta system beter sig korrekt behövs ett schema som uppfyller vissa krav. Att beräkna fram ett schema för storskaliga och praktiskt relevanta instanser är svårt och ur ett optimeringsperspektiv är det intressant att utvärdera hur olika algoritmval kan påverka beräkningsprestanda och lösningstider. Denna avhandling bidrar till detta genom att introducera och utvärdera olika metoder för att beräkna scheman.

Metoderna i avhandlingen bygger på dekompositionsstrategier. Detta innebär att man på ett systematiskt sätt löser flera enklare delproblem för att hitta en lösning till det ursprungliga problemet. I denna avhandling formuleras dessa delproblem antingen som blandade heltalsproblem eller villkorsprogrammeringproblem. Genom att skapa olika typer av delproblem och informationsutbyten dem emellan, introducerar avhandlingen olika metoder som på olika sätt utnyttjar problemstruktur för att få god beräkningsprestanda.

Tre av delprojekten i avhandlingen introducerar metoder för att lösa schemaläggningsproblemet från Saab. Den första metoden bygger på bivillkorsgenerering. Där löses först ett förenklat delproblem, vars lösning sedan utnyttjas för att konstruera ett begränsat delproblem där man söker efter en komplett lösning. Misslyckas detta så adderas villkor till det förenklade problemet som sedan löses om. Denna procedur upprepas tills dess att ett tillåtet schema erhålls eller att man bevisat att det inte går att hitta ett tillåtet schema. I den andra metoden kompletteras bivillkorsgenereringen med en skräddarsydd heuristisk sökstrategi för att effektivt hitta lösningar till det förenklade problemet. Detta görs genom att fixera lösningen i delar av problemet medan man söker efter förbättringar i andra.

Den tredje metoden bygger på en dekompositionsstrategi som kallas logisk-baserad Bendersdekomposition (LBBD). Denna metod snabbas upp av en speciellt framtagen teknik som löser delproblem för att precisera den information som genereras i dekompositionen. Denna teknik genererar och utvärderar samtidigt kvalificerade gissningar för vart lösningar till det kompletta problemet kan hittas. Det fjärde projektet i avhandlingen studerar LBBD i ett bredare perspektiv. I detta projekt jämförs olika metoder för att precisera den information som skickas mellan delproblem i dekompositionsstrategierna genom att tillämpa dem på olika problemklasser. Beräkningsresultaten visar på att det lönar sig att spendera den extra tiden som krävs för att precisera informationen jämfört med att lösa dem som vanligt. 


\section{Acknowledgements}

First I would like to give thanks to my main supervisor Elina Rönnberg. You have shaped my $\mathrm{PhD}$ journey more than anyone else and have by example showed me what it means to be a researcher. Throughout this period, you have not only cared for me as a $\mathrm{PhD}$ student but also as a person and as a colleague. I am the first $\mathrm{PhD}$ student under your main supervision and that is something I always will be proud of. I want to thank my supervisors Kaj Holmberg and Simin Nadjm-Tehrani. Kaj, I will in particular remember your passion for teaching and what I have learned from your $\mathrm{PhD}$ courses. Simin, you allowed me to glimpse into the world of real-time systems and your everyday research environment while taking time when I have had questions. I want to thank my colleagues at the Department of Mathematics for good discussions, help with administration, and providing a good place to work. A particular shout-out to Björn Morén for our "fikas". I want to thank Martin Singull for sparking my interest in research as an undergraduate student.

The contributions of this thesis would have not been possible without Saab and during the last years, my work has been part of a research collaboration between Saab and Linköping University titled "Efficient use of hardware resources in avionic systems". Thanks to all people who have been involved in making this possible. Particular thanks to Andreas Stenberg and Hannes Uppman, who I have worked closely together with and learned a lot from and together with. I want to thank Robert Pettersson and Mats Ekman for technical discussions and giving me feedback on various things through the years. I also want to thank current and past managers, in particular Fredrik Lorentzon, Karolina Bergström, and Tobias Olsson.

I want to thank the Research school in Interdisciplinary Mathematics for funding my research and granting me the possibility to learn about optimisation and real-time system through my $\mathrm{PhD}$ studies. I want to thank the Swedish National Infrastructure for Computing (SNIC) at National Supercomputer Centre (NSC) for providing computational resources.

My life has been blessed by people that I love. Each and every one of you have my thanks. But a special thanks to Tahere for caring and always being kind, and to Ghazaleh, the love of my life, I love you always.

Emil Karlsson, Linköping, 2021 


\section{Contents}

$\begin{array}{lll}\text { Abstract } & \text { iii }\end{array}$

Populärvetenskaplig sammanfattning iv

Acknowledgments v v

Contents vi

List of abbrevations $\quad$ ix

1 Introduction 1

1.1 Outline of the thesis summary . . . . . . . . . . . 2

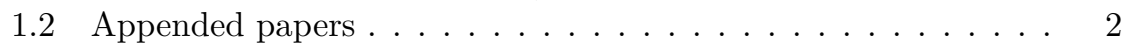

1.3 Author statement . . . . . . . . . . . . . . . . . . 4 4

1.4 Other written work $\ldots \ldots \ldots \ldots \ldots \ldots \ldots$

1.5 Presentations ................... 5

2 Technical background $\quad 7$

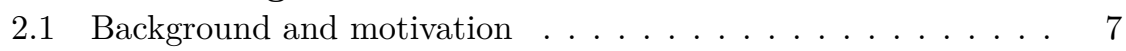

2.2 An avionics scheduling design case $\ldots \ldots \ldots \ldots$

2.3 Instances . . . . . . . . . . . . . . . . . . . 15

3 Mathematical models for single machine scheduling 21

3.1 A single machine scheduling problem with release times and deadlines . . . . . . . . . . . . . . . . . . . 22

3.2 Mixed-integer programming models . . . . . . . . . . . . . 23

3.3 Constraint programming models . . . . . . . . . . . . 32

3.4 Two decomposition schemes for a single machine scheduling

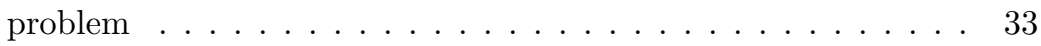

4 Contributions 41

4.1 An avionics scheduling design case . . . . . . . . . . . 41

4.2 Industrially relevant avionics scheduling instances . . . . . . 42

4.3 Decomposition to exploit problem structure . . . . . . . . . 42 
4.4 A matheuristic improvement . . . . . . . . . . . . . . . 43

4.5 Accelerating logic-based Benders decomposition . . . . . . . . 43

$\begin{array}{ll}\text { Bibliography } & 45\end{array}$

$\begin{array}{ll}\text { Paper A } & 51\end{array}$

$\begin{array}{lr}\text { Paper B } & 81\end{array}$

$\begin{array}{lr}\text { Paper C } & 119\end{array}$

$\begin{array}{ll}\text { Paper D } & 151\end{array}$ 



\section{List of abbrevations}

AFDX - Avionics Full-DupleX switched Ethernet

ALNS - Adaptive Large Neighbourhood Search

ARINC - Aeronautical Radio, INC

ASHLEY - Avionics Systems Hosted on a distributed modular electronics Large scal dEmonstrator for multiple tYpe of aircraft

AM - Application Module

CM - Communication Module

CP - Constraint Programming

CN - Communication Network

DFBS - Depth-First Binary Search

DME - Distributed Modular Electronics

EASA - European Aviation Safety Agency

EUROCAE - EURopean Organisation for Civil Aviation Equipment

FAA - Federal Aviation Administration

IMA - Integrated Modular Avionics

LBBD - Logic-Based Benders Decomposition

LP - Linear Programming

MIP - Mixed-Integer Programming

NASA - National Aeronautics and Space Administration

RTCA - Radio Technical Commission for Aeronautics

SCARLETT - SCAlable \& ReconfigurabLe Electronics plaTforms and Tools 



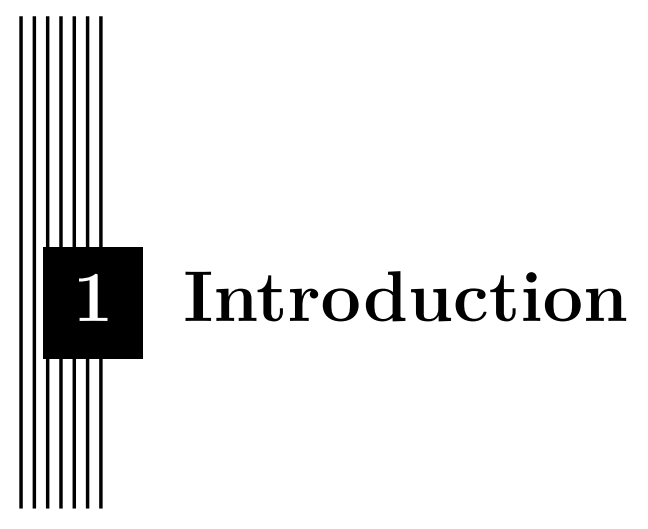

This thesis is about the development of optimisation methods for scheduling of avionic systems. The focus has been on an avionics scheduling design case proposed by Saab Aeronautics. The design case addresses pre-runtime scheduling of a distributed Integrated Modular Avionics (IMA) system and is of interest for future development of aircraft. The scheduling can be categorised as a rich multiprocessor scheduling problem that includes sub-intervals, precedence constraints, and a Communication Network (CN). The problem is of interest for both optimisation and avionics scheduling. However, the contributions of this thesis are within optimisation.

Together, the papers of this thesis provide insights into the structure of this problem and how to exploit it to design efficient solution methods. As part of this, three methods that exploit the problem's structure have been developed to address the computational challenges of solving large-scale instances. These methods combine a wide range of techniques such as constraint generation, Logic-Based Benders Decomposition (LBBD), matheuristics, Adaptive Large Neighbourhood Search (ALNS), Mixed-Integer Programming (MIP) and Constraint Programming (CP). In addition to the focus on the avionics scheduling problem, the computational performance of cut-strengthening approaches for LBBD was evaluated.

This thesis is divided into two parts, a thesis summary followed by appended papers. 


\subsection{Outline of the thesis summary}

This first chapter gives a brief introduction and describes the structure of this thesis. Chapter 2 introduces the avionics scheduling design case. Chapter 3 describes MIP and CP formulations for single machine scheduling and connects them to the decompositions of the appended papers. The last chapter of the thesis, Chapter 4, summarises the contributions of the appended papers. Chapter 2 and Chapter 3 are partly based on the introductory part of my Licentiate thesis [22].

\subsection{Appended papers}

There are four appended papers in this thesis, and they are included in chronological order.

\section{Paper A - An optimisation approach for pre-runtime scheduling of tasks and communication in an integrated modular avionic system}

Paper A introduces an industrially relevant pre-runtime avionic scheduling problem along with a decomposition-based solution approach. The solution approach relies on constraint generation and iteratively solves a master problem and a subproblem using MIP. This procedure is evaluated on three instances provided by Saab.

\section{Publication status}

Paper A has been published in the journal Optimization and Engineering, volume 19, issue 4 in pages 977-1004, 2018.

\section{Authors}

Mathias Blikstad, Tomas Lööw, Emil Karlsson and Elina Rönnberg.

\section{Paper B - A matheuristic approach to large-scale avionic scheduling}

Paper B extends the constraint generation procedure presented in Paper A into a matheuristic. In the matheuristic, the master problem of the constraint generation procedure is solved using an ALNS that iteratively solves MIP models. This work also presents improved MIP-formulations, which impact both the constraint generation procedure and the matheuristic. The constraint generation procedure and the matheuristic are evaluated both on 120 public instances introduced in this paper and on 5 Saab instances. The 
computational results shows that the matheuristic performs better than the constraint generation procedure on large instances.

\section{Publication status}

Paper B has been published in the journal Annals of Operations Research, https://doi.org/10.1007/s10479-020-03608-6, 2020.

\section{Authors}

Emil Karlsson, Elina Rönnberg, Andreas Stenberg and Hannes Uppman.

\section{Paper C - Logic-based Benders decomposition with a partial assignment acceleration technique to solve an avionics scheduling problem}

Paper C introduces a LBBD scheme to solve the avionics scheduling problem. The LBBD scheme involves solving a master problem with a MIP solver and a subproblem with a $\mathrm{CP}$ solver. To enhance the computational performance, we introduce an acceleration technique that extends cut strengthening with a component that tries to construct feasible solutions based on information from the cut strengthening. The computational comparisons show that the LBBD method with the new acceleration technique outperforms the methods in Paper A and Paper B in terms of computational performance and RAM usage.

\section{Authors}

Emil Karlsson and Elina Rönnberg.

\section{Publication status}

Paper $\mathrm{C}$ is a manuscript.

\section{Paper D - Strengthening of feasibility cuts in logic-based Benders decomposition}

Paper D investigates the impact of cut-strengthening approaches for LBBD. Three approaches to cut strengthening, Depth-First Binary Search (DFBS), deletion filter and greedy, are evaluated on three different problems. Computational evaluations are performed for five different instances sets with a total of 2000 instances. The results show that the DFBS cut-strengthening approach, which is adapted from the work on finding irreducible infeasible subsets of constraints, performs best. 


\section{Authors}

Emil Karlsson and Elina Rönnberg.

\section{Publication status}

Paper D has been accepted for publication in the proceedings of the 18th International Conference on the Integration of Constraint Programming, Artificial Intelligence, and Operations Research (CPAIOR 2021).

\subsection{Author statement}

Paper A and Paper B have been co-authored with my main supervisor, Elina Rönnberg, and colleagues from Saab. The latter have contributed to various parts of the research process and especially to the problem formulation and the software development aspects of the implementations. I have contributed to the mathematical modelling, the development of the solution methods, including implementation and testing, and to the preparation of the manuscripts. I was responsible for creating the public avionics scheduling instances and I have implemented the scripts that generate the instances. In Papers C and D, I performed all implementation and testing, while the other parts of the research were done together with my main supervisor, who is a co-author of the papers. The author order of Papers A-D are based on the surnames of the authors and do not reflect the individual contributions of the authors.

\subsection{Other written work}

During my doctoral studies, I have also written or co-authored the following work.

- Emil Karlsson and Elina Rönnberg. Explicit modelling of multiple intervals in a constraint generation procedure for multiprocessor scheduling. Editors: N. Kliewer, J.F. Ehmke, and R. Borndörfer. Operations Research Proceedings 2017 (OR2017), by Springer International Publishing, pages 567-572, 2017. (peer-reviewed)

- Emil Karlsson. Optimisation-based scheduling of an avionic system. Linköping University. Technical faculity, Licentiate Thesis, number $1844,2019$.

- Emil Karlsson and Elina Rönnberg. Description of instances for an avionics scheduling problem. Report, accessible at https:// gitlab.liu.se/eliro15/avionics_inst/-/blob/master/instance_ description.pdf, 2020. 


\subsection{Presentations}

During my doctoral studies I have attended and made presentations at the following conferences.

- International Conference on Operations Research 2017 (OR2017). This conference took place in Berlin, Germany, in 6-8 September 2017. I presented the work of publication OR2017.

- Svenska operationsanalyskonferensen 2017 (SOAK2017). This conference took place in Linköping, Sweden, in 19-20 October 2017. I presented the work of publication OR2017.

- European Conference on Operational Research 2019 (EURO2019). This conference took place in Dublin, Ireland, in 23-26 June 2019. I presented the work of Paper B.

- Aerospace Technology Congress 2019 (ATC2019). This conference took place in Stockholm, Sweden, in 8-9 October 2019. I presented the work of Paper A and Paper B.

- Svenska operationsanalyskonferensen 2019 (SOAK2019). This conference took place in Nyköping, Sweden, in 23-24 October 2019. I presented the work of Paper B.

I have also been accepted to present the work of Paper D at the 18th International Conference on the Integration of Constraint Programming, Artificial Intelligence, and Operations Research (CPAIOR 2021), 5-8 July 2021. 



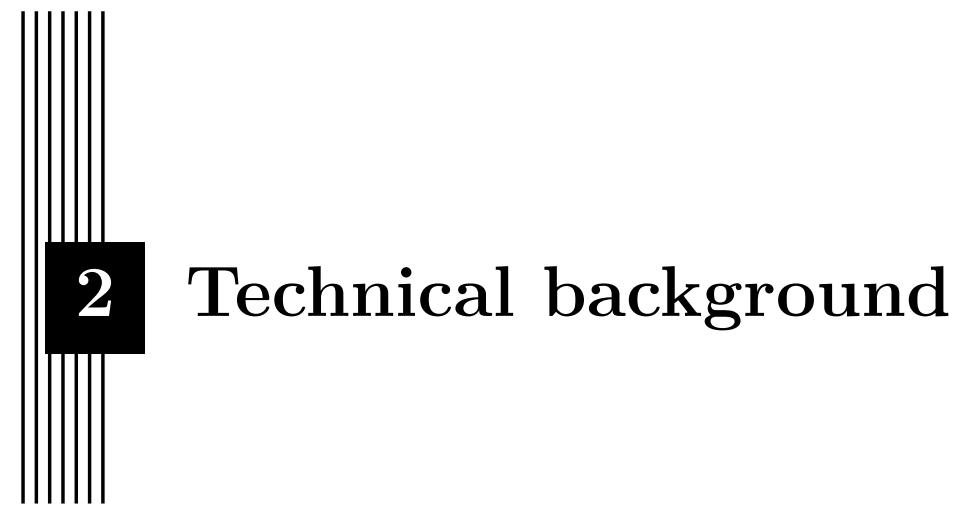

This chapter introduces the avionics scheduling design case that is the focus of the work in this thesis. The chapter includes a brief description of the background to the design case, an explanation of the optimisation problem, and a description of the instances used in this thesis.

\subsection{Background and motivation}

An aircraft is often developed and maintained through many years (even decades) and receives regular updates of software and hardware during its lifetime. Guidelines on how to develop safe and reliable aircraft are put together through the collaboration between different actors: aircraft manufacturers such as Saab, Boeing, and Airbus; agencies such as the European Aviation Safety Agency (EASA) in Europe and the Federal Aviation Administration (FAA) in the USA; and organisations such as the Radio Technical Commission for Aeronautics (RTCA) and the EURopean Organisation for Civil Aviation Equipment (EUROCAE). RTCA and EUROCAE have for example published guidelines for the development of software, DO-178C [36] (also called ED-12C [12]), and the development of hardware, DO-254 [37] (also called ED-80 [13]), for aircraft.

In today's aircraft, many avionic functions such as steering, navigation, and displays, use software. However, when computers first were used in aircraft, each avionic function with electronic components was implemented on a dedicated computer that was connected to the sensors and actuators that 
the avionic function required. If avionic functions needed to interact with each other, e.g. by reading the value from the same sensor, direct cables were drawn between their computers. Avionic systems of this kind are called federated systems. The technical growth was fast, and the federated systems went through several transitions from analogue circuits to digital circuits into more sophisticated processors. Meanwhile, the methods for transferring data transitioned from direct cables to serial links into methods that can guarantee reliable transfer of data between computers, such as Aeronautical Radio, INC (ARINC) 429 and MIL-STD-1553, that were released in the 1970s.

As the number of avionic functions that used software grew, so grew the size and weight of federated systems. Beginning in the late 1980s and early 1990s, a new type of avionic systems emerged, called IMA systems. In an IMA system, multiple avionic functions can be implemented on the same hardware, which enables a more efficient usage of computing resources than in federated systems. Standardisation efforts started early to establish technologies that together with an IMA design can be applied to form a complete system. An example of such is the ARINC 653 standard for space and time partitioning within operating systems, that allows software of various levels of criticality to be implemented on the same hardware. In an ARINC 653-compliant operating system, it is possible to host software applications with various levels of criticality on the same hardware. Another example of standardisation is that the first methods and standards that use Ethernet to transfer data between computers in avionic system, such as the ARINC 664 standard, the Avionics Full-DupleX Switched Ethernet (AFDX) developed by Airbus, and time-triggered Ethernet, were released. After a few years, guidelines on how to develop and certify IMA systems, see for example DO-297 by RTCA [38] (also called ED-124 [14]), were published. The type of IMA systems that emerged during this time were later referred to as the $1^{\text {st }}$ generation of IMA systems. Particularly, the $1^{\text {st }}$ generation of IMA systems demonstrated benefits when applied to a limited number of aircraft functions. However, to obtain a fully integrated IMA system, additional work needed to be done.

During the last two decades, there has been large research projects that investigate IMA systems that better support incremental development and verification of software and hardware. In Europe, the SCAlable \& ReconfigurabLe Electronics plaTforms and Tools (SCARLETT) project and the Avionics Systems Hosted on a distributed modular electronics Large scal dEmonstrator for multiple tYpe of aircraft (ASHLEY) project examined the next generation of IMA systems, while in the USA, the National Aeronautics and Space Administration (NASA) Orion Space program, investigated the next generation of avionic systems for future space travels. A $2^{\text {nd }}$ generation of IMA systems, sometimes called Distributed Modular Electronics (DME)-based IMA systems, was suggested as part of these research projects, see for example [15]. The intention of a DME-based IMA system is to support all aircraft functions in a fully integrated system. With e.g., standardised hardware and standard- 
ised communication, the system is intended to be more scalable and adaptable than the $1^{\text {st }}$ generation of IMA systems. A DME-based IMA system can have multiple IMA units within the same avionic system. In a system with multiple IMA units, each IMA unit is connected to a module dedicated to communication that can transfer data to another part of the avionic system through a shared aircraft communication network. A DME-based IMA system has the advantages of an IMA system, such as better resource utilisation, while allowing for more aircraft functions to be incorporated into the system.

As federated systems struggle with resource usage and weights, there are also challenges with distributed IMA systems. A disadvantage of creating a complex avionic system is that, as the system grows increasingly complex, so does the challenge of ensuring that software applications get sufficient computing and network communication resources. This increases the complexity in design and verification with respect to fault containment and scheduling [45]. This thesis focuses on the pre-runtime scheduling of an advanced distributed IMA system of interest for future avionic systems. The distributed IMA system contains physically disjoint subsystems that each has a module dedicated to communication. These are in turn connected to a type of shared time-triggered Ethernet network. The scheduling problem is formalised in the avionics scheduling design case and is part of assuring that the timing requirements of the underlying avionic system are fulfilled.

\subsection{An avionics scheduling design case}

The purpose of the avionics scheduling problem is to construct a schedule that can be used in a periodic avionic system. One repetition of the avionic system is called a major frame, and during each such major frame the same schedule is used by a dispatcher to start tasks and send messages. Instances of the avionics scheduling problem are constructed so that the timing requirements of the underlying system will be respected if the system starts tasks and sends messages according to a feasible schedule. The schedule that needs to be constructed is about one second long and has a time resolution in nanoseconds.

The avionics scheduling problem was first introduced in Paper A. There, the problem statement contains a technical restriction, that simplifies the modelling, related to the sending and receiving of messages in the network. The restriction was removed from the problem statement in [40], which introduced how these technical requirements can be handled. A variant of the problem formulation was also introduced in [23], in which some requirements from the problem formulation in Paper A were expressed through subintervals. In Paper B, the problem formulations in Paper A, in [23], and in [40] were combined into a united problem statement. In this section, the avionics scheduling problem, as studied in Paper B and Paper C, is stated as 


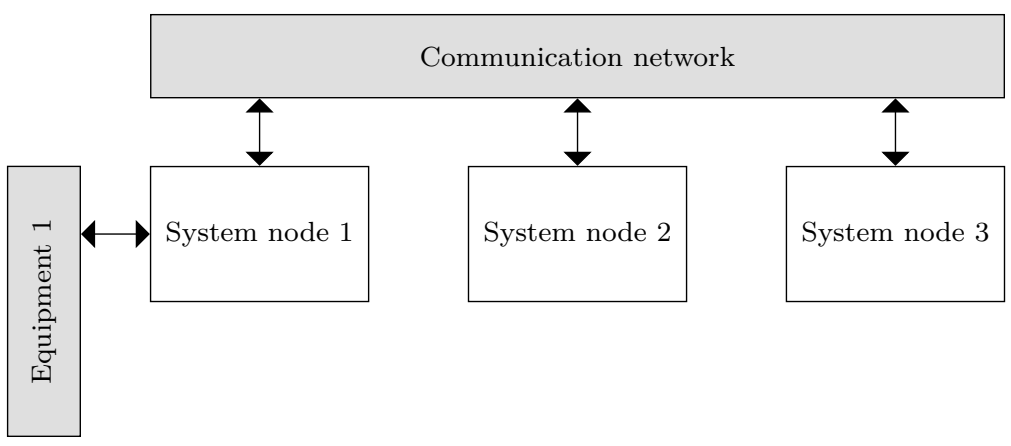

Figure 2.1: An overview of an avionic system with three system nodes and one equipment

an optimisation problem without focusing on its technical background. For a technical background, see the introductory parts of Paper A and Paper B.

\subsubsection{The components of a schedule}

The avionics scheduling problem is a feasibility problem and therefore it does not include an objective function. Instead, the challenge is to construct a schedule where

- each message is assigned a communication slot, and

- each task is assigned a start time,

that is feasible with respect to certain constraints, or to prove that no such schedule exists.

\subsubsection{Task scheduling}

An instance of the avionics scheduling problem contains a set of system nodes. Each system node consists of one Communication Module (CM) and at least one Application Module (AM). On each module, there is a set of tasks that must be scheduled. The CMs are connected to a $\mathrm{CN}$ where messages can be sent between system nodes in time-separated communication slots. Each message is sent from a single CM to a set of receiving CMs. External equipment can also be connected to a system node via its CM. An avionic system with three system nodes, a $\mathrm{CN}$, and an external equipment is illustrated in Figure 2.1 and an example of a system node is illustrated in Figure 2.2.

On each AM, ARINC 653 partitions, which are represented as tasks, need to be scheduled. Finding a schedule for an AM is about finding start times for its partitions so that the partitions' windows of execution does not overlap. Each partition comprises one or more processes that are pre-emptable. 


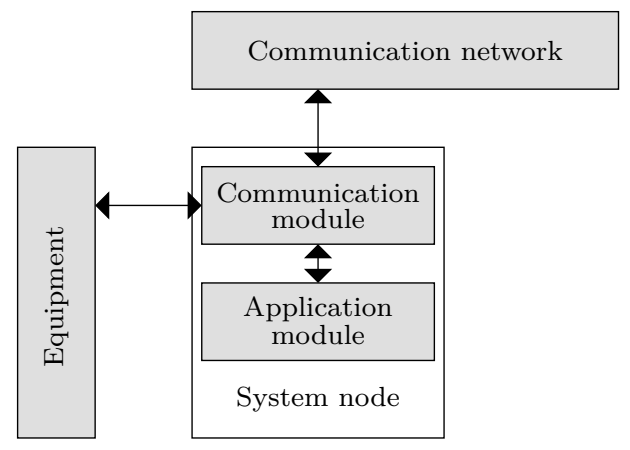

Figure 2.2: A detailed illustration of a system node that contains one application module and one communication module that is connected to an equipment

The processes within a partition are not part of the avionics scheduling problem and are instead scheduled at runtime according to a priority-based ratemonotonic scheduling algorithm.

Each CM is responsible for the communication of its system node. This involves communication within the system node, communication with external equipment and communication with other system nodes through the CN. These acts of communicating are abstracted as tasks, messages, and constraints in instances of the avionics scheduling problem.

The tasks in the system are performed periodically. The tasks on a CM have the same period as the length of the major frame, but the tasks on an AM are performed 64 times within a major frame. When defining precedence constraints between tasks on different modules, a specific occurrence of a task within a major frame is referred to by the term task instance.

Each task in an instance of the avionics scheduling problem is associated with a set of intervals of time, called sub-intervals, where it can start. For a schedule to be feasible, each task must start within one of its sub-intervals. Each task must also be scheduled for the duration of its execution requirement without overlapping any other task on its assigned module. For an AM, this is typically no challenge since AMs have few tasks with long execution requirements. But for a CM, this is typically a challenge since CMs have many tasks with short execution requirements.

\subsubsection{Precedence constraints between tasks}

In an instance of the avionics scheduling problem, there exists two types of precedence constraints between tasks. The two types of constraints are called dependencies and chains. A dependency restricts the duration between the start of a task and the start of the next occurrence of another task. 


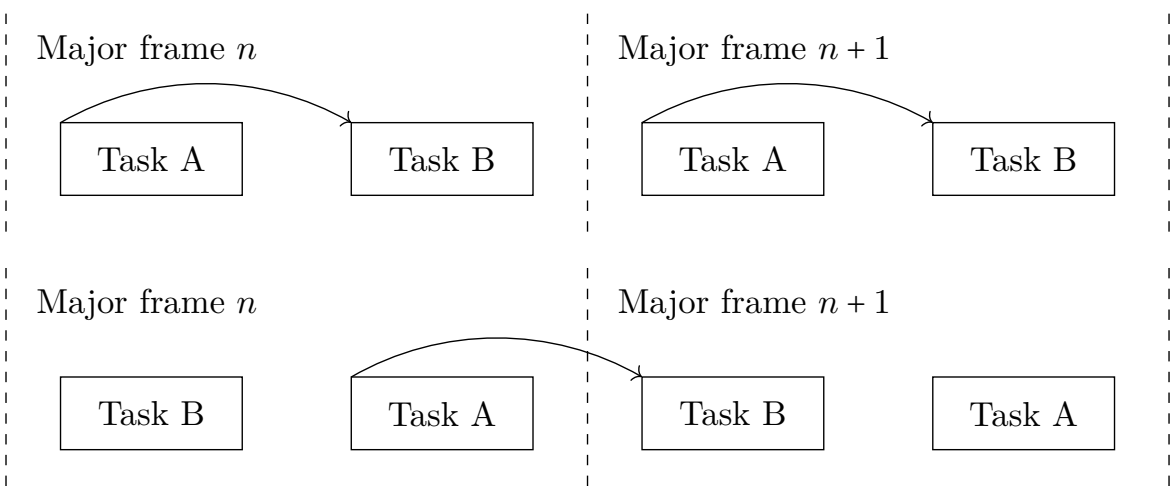

Figure 2.3: Examples of the two ways in which the duration between the tasks of a dependency can be measured depending on their order in a major frame

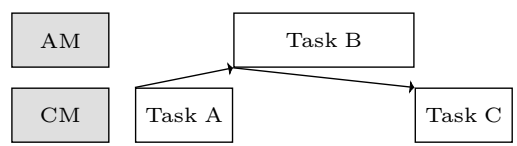

Figure 2.4: A common communication pattern between an AM and a CM that includes three tasks and two dependencies. A dependency between task A and task B ensures that task A on the CM finishes at the same time as task B on the AM starts. A second dependency between task B and task C ensures that task $\mathrm{C}$ on the $\mathrm{CM}$ starts when task $\mathrm{B}$ on the $\mathrm{AM}$ finishes

Note that the start of a task and the start of the next occurrence of another task in a dependency can occur in different major frames, as illustrated in Figure 2.3. If a dependency connects a task from an AM with a task from a $\mathrm{CM}$, the dependency measures its duration from or to the start of a specific task instance of the AM task in a major frame.

In instances of the avionics scheduling problem, there are some dependencies that require a fixed length between the start of its two tasks. These dependencies have to do with the communication within a system node and connect a task instance on an AM with a task on a CM. An example of a common combination of two such dependencies is illustrated in Figure 2.4.

A chain enforces a series of tasks (or task instances if a task is on an AM) to be performed in a given order within the length of a major frame. This means that the cyclic order between the tasks is restricted. Worth noting is that between each adjacent pair of tasks in a chain, there is always a dependency. An illustration of a chain is found in Figure 2.5. 


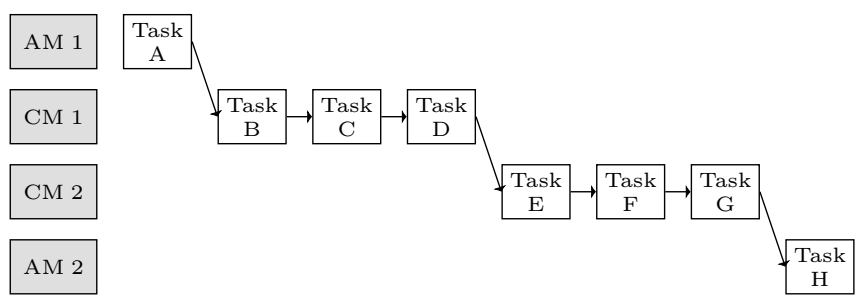

Figure 2.5: Example of a chain with tasks A, B, C, D, E, F, G, and H

\subsubsection{Communication network scheduling}

The $\mathrm{CN}$ is a time-triggered Ethernet where messages are sent in timeseparated communication slots. Each communication slot on the CN uses the network for a specific interval of time and has a certain capacity for data. Each message that shall be sent on the $\mathrm{CN}$ has a certain size and must be assigned to one communication slot. For a schedule to be feasible, each communication slot must have a data capacity that exceeds or is equal to the combined size of the messages assigned to it.

A challenging aspect of the avionics scheduling problem is the interaction between messages and tasks. It is challenging since it is possible to reduce the execution requirements for certain tasks involved with sending and receiving messages by assigning their messages to the same communication slot. Also, assigning messages to the same communication slot puts additional restrictions on how the tasks involved with sending and receiving the messages can be scheduled. Hence, deciding if messages should be assigned to the same communication slot or not is a trade-off between the restricted constraints on start times of involved tasks and reduced execution requirements of the involved tasks. Below, these details are described.

There are four types of tasks involved in sending and receiving a message on a $\mathrm{CN}$ and these are performed in a particular order on the involved CMs. On the sending CM, there is first a task that prepares the message and then a task that sends the message. On each receiving CM, there is first a task that dequeues the message and then a task that reads the data. The messages themselves must be sent in time slots on the $\mathrm{CN}$ which puts requirements on the tasks involved in sending and receiving the message. Figure 2.6 gives an illustration of sending and receiving two messages.

If two or more messages are sent in the same slot, their tasks of the same type on a CM must be merged. This means that the tasks are performed in immediate succession in a schedule and all but one of the tasks are performed with reduced execution requirements. The length of the execution requirement that can be removed from a task is called initialisation time and depends on the CM the task is performed on and the task's type. Figure 2.7 illustrates 


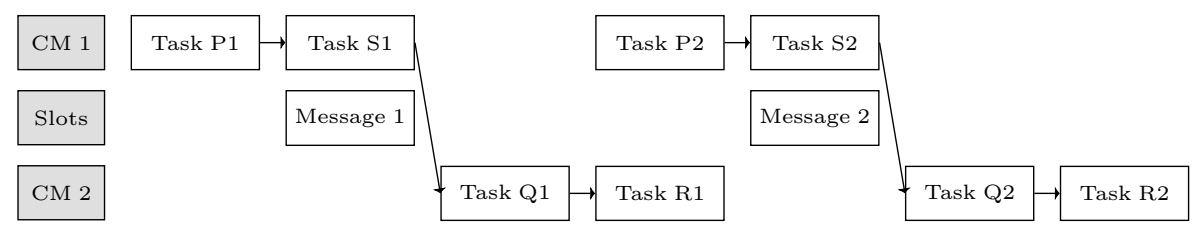

Figure 2.6: An illustration of two messages, message 1 and message 2, and their involved tasks P1, S1, Q1 and R1 for message 1 and P2, S2, Q2 and R2 for message 2

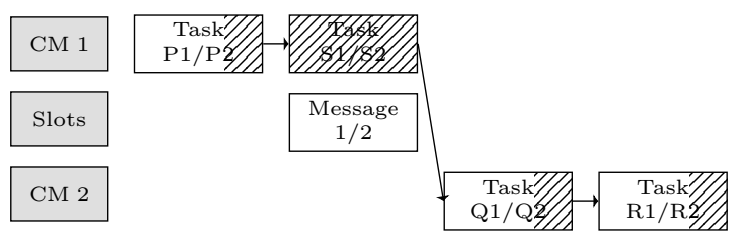

Figure 2.7: Example of where two messages are sent in the same slot and the execution requirements of some tasks involved in sending and receiving them are reduced. The part of the execution requirement of tasks that has been saved compared to if the messages were assigned to different communication slots are patterned grey

two messages and the tasks involved in sending and receiving them when the two messages are assigned to the same communication slot.

The tasks on CMs that send messages have additional constraints on them. If a message is assigned to a communication slot, the task that sends the messages must start at a given time that depends on the communication slot that its message was assigned to. Each communication slot on the $\mathrm{CN}$ has its unique time for where tasks that send messages must start. This means that assigning a message to a communication slot is equivalent to determining in which sub-interval the task that sends the message shall be performed if the task's sub-intervals are properly pre-processed. Another property of the tasks that send messages is that their initialisation time is the same length as their execution requirement. Hence, two tasks on the same CM that sends messages must be started on the same time if their messages are assigned to the same communication slot. This is possible since one of the tasks will have its execution requirement set to zero in this case.

There are also requirements on the tasks that dequeue messages. If a message is assigned to a communication slot, the tasks that dequeue the message must be scheduled within an interval of time that depends on the communication slot its message was assigned to. Furthermore, on each CM, the tasks 
that dequeue messages must be performed in the same order as their messages are sent.

\subsection{Instances}

In Papers $\mathrm{A}-\mathrm{C}$, different instances of the avionics scheduling problem have been used to evaluate the computational performance of the solution strategies that have been developed. This section describes those instances and presents some of their characteristics.

\subsubsection{Instance characteristics}

To run an avionic system, even without any software applications or communication, there are several activities that must be performed, such as accessing sensors and actuators, assuring synchronisation between modules, and establish contact with external equipment and networks. For instances of the avionics scheduling problem, many of these activities appear as tasks and messages that, respectively, must be performed or sent within specific time intervals. There are also dependencies and chains that originate from timerelated and order-related requirements on these activities. The number of tasks, messages, dependencies, and chains that originate from these activities are correlated to the number of $\mathrm{AMs}, \mathrm{CMs}$, and external equipment in the avionic system, but does not increase when additional communication or partitions are added to the avionic system.

Each AM in the underlying avionic system of the avionics scheduling problem runs an ARINC 653 operating system. As mentioned, the partitions of an AM are represented as tasks and are part of the avionics scheduling problem. Even though the processes that constitute partitions are not part of the avionics scheduling problem, the transfer of data between processes is the origin of many of the tasks, dependencies, messages, and chains in instances of the avionic scheduling problem. This is because most communication in the avionic system take place between processes located on the same or on different system nodes.

Almost all chains in instances of the avionics scheduling problem originate from transfers of data between two (or more) parts of the avionic system. The tasks, messages and constraints that originate from communication are rarely tight in isolation, but to schedule the involved tasks and messages together with the tasks and messages that must be performed or sent within specific time intervals is challenging. To give an idea of how communication within the avionic system is related to tasks, messages, dependencies, and chains, we give two examples of chains below.

The first example is a chain of tasks that realises a data transfer between a process in a partition and another process in another partition in a different system node. To transfer the data, eleven tasks must be performed in a 
1. Task (partition) outputs data

2. Task collects data from the AM

3. Task handles and routes the data within the CM

4. Task prepares the message on the $\mathrm{CN}$

5. Task sends the message on the $\mathrm{CN}$

[ Message is sent in a communication slot on the CN ]

6. Task dequeues the message from the $\mathrm{CN}$

7. Task reads the data from the message

8. Task handles and routes data within the CM

9. Task readies data for transfer to the AM

10. Task transfers data to the AM

11. Task (partition) uses the data

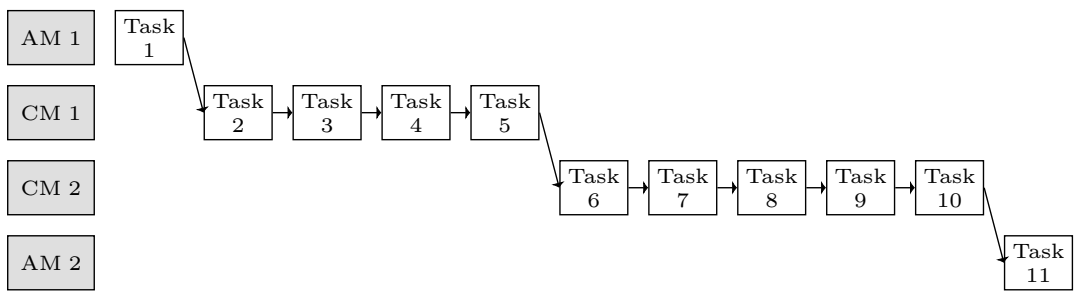

Figure 2.8: Description and illustration of example chain 1

correct order and a message must be sent on the CN. To ensure the correct order between tasks, there is, besides a chain, one dependency between each pair of adjacent tasks in the chain. This chain, its involved tasks and the message that is sent on the $\mathrm{CN}$ as part of the data transfer are illustrated and described in Figure 2.8.

The second example is a chain of tasks that realises a data transfer between a process in a partition and an external equipment connected to the same system node as the partition. To transfer the data, four tasks must be performed in a correct order. To ensure the correct order, there is, besides a chain, one dependency between each adjacent pair of tasks in the chain. This chain and its involved tasks are illustrated and described in Figure 2.9. 


1. Task (partition) outputs data
2. Task collects data from the AM
3. Task handles and routes data within the CM
3. Th
4. Task transfers data to an external equipment

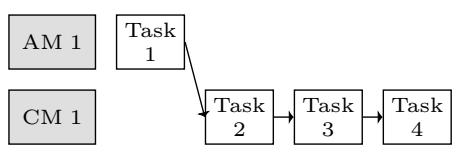

Figure 2.9: Description and illustration of example chain 2

\begin{tabular}{lrrrrr}
\hline Name & Tasks & Messages & Dependencies & Chains & Nodes \\
\hline Instance I & 6538 & 64 & 1088 & 256 & 2 \\
Instance II & 14,186 & 96 & 9784 & 384 & 5 \\
Instance III & 19,919 & 96 & 12,616 & 928 & 7 \\
Instance IV & 20,461 & 240 & 30,245 & 18,870 & 2 \\
Instance V & 26,268 & 1032 & 30,374 & 18,258 & 3 \\
Instance VI & 45,026 & 2616 & 48,862 & 27,446 & 8 \\
\hline
\end{tabular}

Table 2.1: The number of tasks, dependencies, messages, chains, and system nodes of Instances I-VI provided by Saab

\subsubsection{Saab instances}

In Paper A and Paper B, instances directly provided by Saab were used to evaluate the computational performance of the introduced methods. These instances contain specific properties that are important for Saab, and they are not publicly available. Instances I-III were introduced in Paper A, while Instances IV-VI were introduced in Paper B. The number of tasks, messages, dependencies, chains, and system nodes of Instances I-VI can be found in Table 2.1. Among Instances I-VI, Instance VI is the most challenging due to its large number of tasks and messages.

\subsubsection{Public instances}

Public instances of the avionics scheduling problem were developed together with engineers from Saab to be industrially relevant and they were first introduced in Paper B. The public instances have been used for computational 


\begin{tabular}{lrrrrr}
\hline Category & Tasks & Messages & Dependencies & Chains & Nodes \\
\hline Category A & 4932 & 172 & 9516 & 7320 & 2.0 \\
Category B & 11,699 & 447 & 22,170 & 8808 & 2.0 \\
Category C & 20,037 & 908 & 37,707 & 20423 & 4.1 \\
Category D & 41,655 & 1923 & 79,503 & 47085 & 8.2 \\
\hline
\end{tabular}

Table 2.2: The average number of tasks, messages, dependencies, chains, and system nodes of Categories A-D of the public instances

evaluation of the methods introduced in Paper B and Paper C and are available at [2].

There are currently four categories of public instances with 30 instances in each category. Table 2.2 provides, for each instance category, the average number of tasks, messages, dependencies, chains, and system nodes. The relationships between the number of tasks, dependencies, messages, chains, and system nodes of the instance categories are illustrated in Figure 2.10.

Category A contains instances of about the same size and difficulty as Instance I from the Saab instances. Both Categories B and C contain instances designed to be of medium difficulty. Instances in Category D are constructed to be challenging due to the large numbers of tasks and messages. The largest instance in Categories A-D contains 54,731 tasks and 2530 messages. Instances in Category B-D have the important characteristic that they are unbalanced with respect to that certain $\mathrm{CMs}$ are assigned more tasks and handle more messages compared to other CMs in the system. The instances in Category A are not given this property, to keep them simpler. 

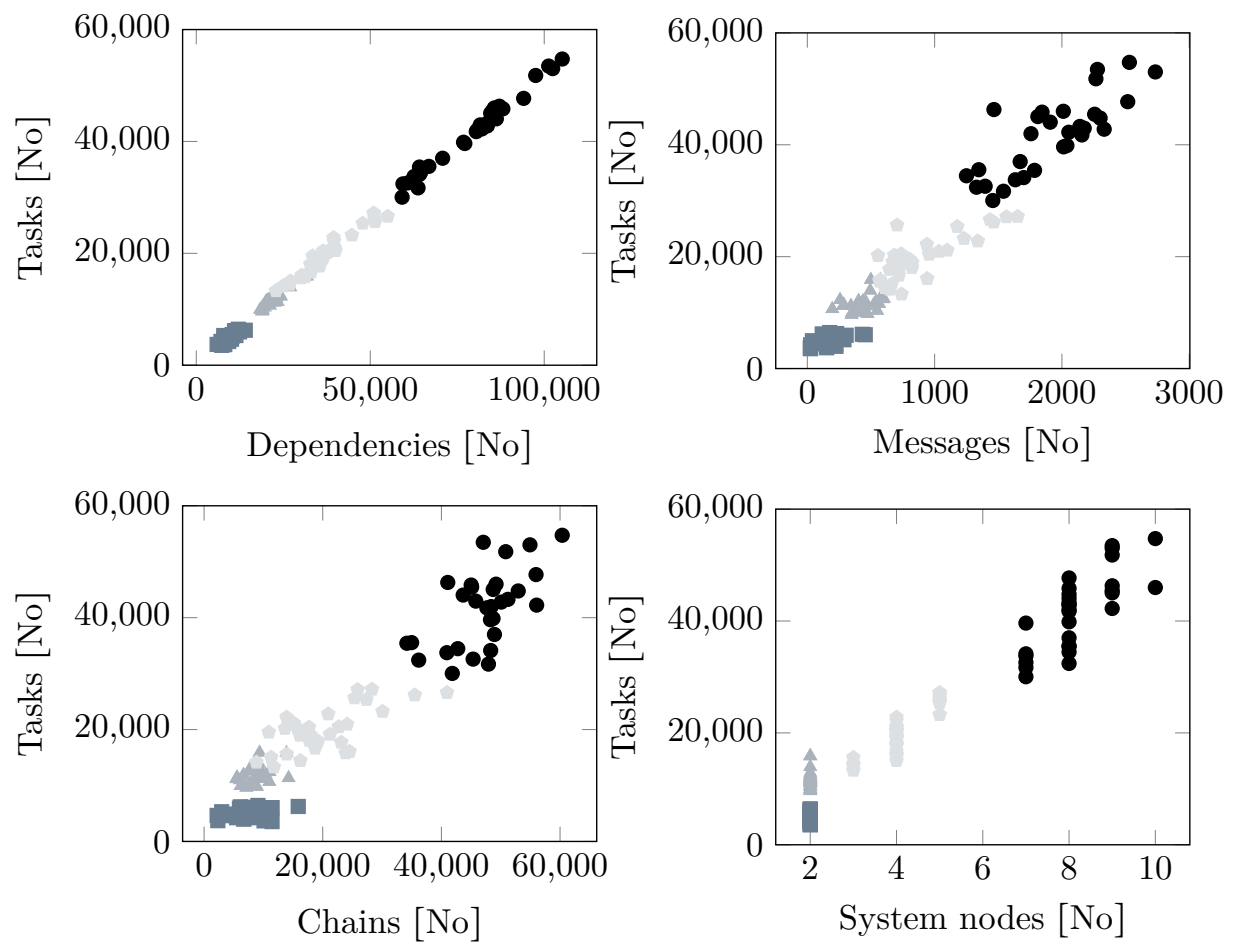

- Category A $\triangle$ Category B $\quad$ Category C Category D

Figure 2.10: Relations between the number of tasks, dependencies, messages, chains, and system nodes in the public instances 



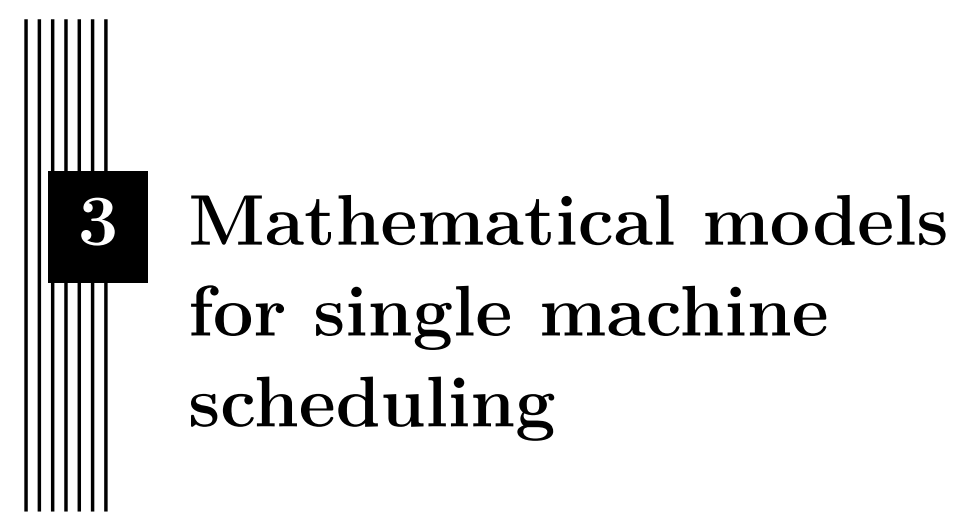

A core aspect of many large-scale scheduling problems is to sequence tasks. The most fundamental type of such scheduling problems are the so-called single machine scheduling problems. In a single machine scheduling problem, a set of tasks (jobs or activities) must be scheduled without overlap on a single processor (machine or resource). Typically, there are also additional constraints and possibly an objective function to consider. Such additions can greatly influence the computational complexity of a problem.

To sequence tasks is often an important part of complex scheduling problems. To solve such problems efficiently, the modelling of sequencing parts is often essential. As such, mathematical models for single machine scheduling problems have been extensively studied. In this chapter, we survey a variety of MIP formulations along with a CP formulation for a single machine scheduling problem with release times and deadlines. To relate these to the work in this thesis, we also present two decomposition schemes, inspired by the decompositions in Paper A-D, for a single machine scheduling problem with multiple time-windows and sequence-dependent setup times. 


\subsection{A single machine scheduling problem with release times and deadlines}

In this section, we introduce the terminology and notation for the single machine scheduling problem with release times and deadlines. In the following sections, we will introduce different mathematical models for this problem.

Let $\mathcal{I}$ denote a set of tasks that must be scheduled without pre-emption on a single machine that can handle at most one task at a time. Associated with each task $i \in \mathcal{I}$, are a release time $r_{i}$ and a deadline $d_{i}$, specifying an interval $\left[r_{i}, d_{i}\right]$ wherein the task must be processed. The processing time (or duration) of task $i \in \mathcal{I}$ is denoted by $p_{i}$. The aim is to evaluate if there exist a feasible schedule (and if one such exists; give it) that respect the release time and deadline of each task. Using the $(\alpha|\beta| \gamma)$ classification scheme in [30], a slight extension of the one originally introduced in [18], this problem can is classified as $\left(1\left|r_{j}, \bar{d}_{j}\right|-\right)$. In [16], this problem was proven to be NP hard for arbitrary release times, deadlines, and processing times.

\subsubsection{A note on computational complexity}

As with many classes of discrete optimisation problems, the computational complexity of single machine scheduling problems are greatly impacted by details. A detail can be the difference between a single machine scheduling problem that is NP hard and one that is solvable in polynomial time.

In the single machine scheduling problem with release times and deadlines, tasks are scheduled without pre-emption, without any objective function but with hard constraints on their release times and deadlines. In this problem, the release times, deadlines, and processing times are assumed to be task unique. If the deadlines would be equal for all tasks $\left(1\left|r_{j}, \bar{d}_{j}=d\right|-\right)$ or the release times would be equal for all tasks $\left(1\left|\bar{d}_{j}\right|-\right)$, the single machine scheduling problem with release times and deadlines can be solved in polynomial time using the earliest deadline first algorithm [21]. The earliest deadline first algorithm is sometimes also called Jackson's rule. If release times and deadlines are task-unique, but pre-emption of tasks was allowed $\left(1 \mid r_{j}, \bar{d}_{j}\right.$, pmtm $\left.\mid-\right)$, the extended Jackson's rule [20] can solve the problem in polynomial time. If instead the processing times for tasks would be equal $\left(1\left|r_{j}, \bar{d}_{j}, p_{j}=p\right|-\right)$, an algorithm presented in [42] can solve the problem in polynomial time.

Many single machine scheduling problems does not have hard deadlines for its tasks, but rather a due date that can be violated with a cost. When including due dates, it is common to add an objective function to the single machine scheduling problem. In increasing order of difficulty with respect to computational complexity, three common objective functions for the single machine scheduling problems with release times are to minimise the maximum due date violation, called maximum lateness $\left(1\left|r_{j}\right| L_{\max }\right)$, minimise the number of tasks that violate the due date, called minimise the number of tardy 
tasks ( $1\left|r_{j}\right| U_{j}$ ), and to minimise the sum of the due date violations, called tardiness $\left(1\left|r_{j}\right| T_{j}\right)$. Essentially, the constraints and the objective function used for a problem can greatly influence its computational complexity.

\subsection{Mixed-integer programming models}

Shortly after the introduction of MIP [17], three MIP approaches for scheduling problems were introduced. The three approaches differed in their choice of main binary decision variables that are either time oriented, position oriented, or order oriented. Different formulations based on time-oriented, positionoriented, and order-oriented binary decision variables are presented in this section.

\subsubsection{Time-oriented formulations}

In this section, we present three MIP formulations where the main binary decision variables are time oriented. For such formulations, typically the number of binary variables increases with the length of the scheduling horizon.

\section{Time-indexed formulation}

The time-indexed approach divides the scheduling horizon $T=\max _{i \in \mathcal{I}} d_{i}$ into unit-size time steps and use binary decision variables to indicate whether a particular task starts at a particular time step or not. This requires that all processing times of tasks are rational or preferably even integer.

Introduce for task $i \in \mathcal{I}$, and time step $t, t=0, \ldots, T$, the binary variable

$$
x_{i t}= \begin{cases}1, & \text { if task } i \text { starts in time step } t \\ 0, & \text { otherwise }\end{cases}
$$

By finding a solution that obey

$$
\begin{gathered}
\sum_{t^{\prime}=0}^{T-p_{i}+1} x_{i t^{\prime}}=1, \quad i \in \mathcal{I}, \\
\sum_{i \in \mathcal{I}} \sum_{t^{\prime}=t-p_{i}+1}^{t} x_{i t^{\prime}} \leq 1, \quad t=0, \ldots, T, \\
\sum_{t^{\prime}=0}^{r_{i}-1} x_{i t^{\prime}}=0, \quad i \in \mathcal{I}, \\
\sum_{t^{\prime}=d_{i}-p_{i}+1}^{T} x_{i t^{\prime}}=0, \quad i \in \mathcal{I},
\end{gathered}
$$

we have a feasible schedule for the single machine scheduling problem with release times and deadlines. Constraints (3.2) assign each task a time step to 
start in. Constraints (3.3) prevent processing of more than one task in a time step. Constraints (3.4)-(3.5) make sure that each task respects its release date and deadline.

The time-indexed formulation was introduced in [8] for what we today would call a machine scheduling problem. In this initial formulation, the binary variables used were slightly different and instead indicated whether or not a task should be processed at a given time. Another version was used some years later in [34], in which the binary variables indicated if a task finished at a certain time step or not. In the early 1990s, theoretical properties and valid inequalities for time-indexed formulations were first studied in [11]. In [43], further valid inequalities based on knapsack relaxations were presented along with a procedure to generate such valid inequalities. This procedure was used in a cutting plane framework that was tested on single machine scheduling instances. Additional valid inqualities were published in [1], that focused on inequalities with the right-hand side equal to 1 and 2 .

\section{Interval-indexed formulation}

The interval-indexed formulation was introduced in [5]. In this formulation, the scheduling horizon is partitioned into intervals. The idea behind the formulation is to use variables to keep track of if each task has started in an interval or earlier, if each task has completed within an interval or earlier, and measure the idle time for each interval. With such variables, it is possible to add constraints to ensure that tasks do not overlap, and that release times and deadlines must be obeyed.

Let $t_{0}<t_{1}<\cdots<t_{Q}$ denote the start and end points of a partition of the scheduling horizon with $Q$ intervals. The start point and end point of interval $q=1, \ldots, Q$, are $t_{q-1}$ and $t_{q}$, respectively. To handle release times and deadlines in tasks, the partition of the scheduling horizon must be valid. A partition of the scheduling horizon is considered valid if every release time in a task is the start of an interval and every deadline in a task is the end of an interval. Below, we assume that a valid partition of the scheduling horizon is used.

To construct the model, some additional notation is needed. For each interval $q=1, \ldots, Q$, let $\mathcal{I}_{q}^{\mathrm{S}}=\left\{i \in \mathcal{I}: t_{q}-t_{q-1} \geq p_{i}\right\}$ denote the set of tasks with a processing time less than or equal to the length of the interval. For each interval $q=1, \ldots, Q$, let $\mathcal{I}_{q}^{\mathrm{B}}=\left\{i \in \mathcal{I}: t_{q}-t_{q-1}<p_{i}\right\}$ denote the set of tasks with a processing time larger than the length of the interval.

For each task $i \in \mathcal{I}$ and interval $q=1, \ldots, Q$, introduce the binary variables

$$
z_{i q}^{\mathrm{S}}= \begin{cases}1, & \text { if task } i \text { starts in interval } q \text { or earlier } \\ 0, & \text { otherwise }\end{cases}
$$


and

$$
z_{i q}^{\mathrm{E}}= \begin{cases}1, & \text { if task } i \text { is completed in interval } q \text { or earlier } \\ 0, & \text { otherwise. }\end{cases}
$$

We say that a task $i \in \mathcal{I}$ is started in interval $q=1 \ldots, Q$, if it starts within the interval $\left[t_{q-1}, t_{q}\right)$. We say that a task $i \in \mathcal{I}$ is completed in interval $q=1 \ldots, Q$, if it is completed within the interval $\left(t_{q-1}, t_{q}\right]$. Also introduce for each interval $q=1, \ldots, Q$, the continuous variable

$$
u_{q}^{\text {idle }}=\text { the idle time of interval } q .
$$

The authors of [5] prove that the tasks can always be ordered in a specific way that makes the idle time for an interval well defined. This provides the means to find feasible start times for tasks given a solution to the formulation below.

By solving

$$
\begin{gathered}
z_{i, q-1}^{\mathrm{E}} \leq z_{i q}^{\mathrm{E}}, \quad q=1, \ldots, Q, i \in \mathcal{I}, \\
z_{i, q-1}^{\mathrm{S}} \leq z_{i q}^{\mathrm{S}}, \quad q=1, \ldots, Q, i \in \mathcal{I}, \\
z_{i q}^{\mathrm{E}} \leq z_{i q}^{\mathrm{S}}, \quad q=1, \ldots, Q, i \in \mathcal{I}, \\
z_{i, q-1}^{\mathrm{S}} \leq z_{i, q}^{\mathrm{E}}, \quad i \in \mathcal{I}_{q}^{\mathrm{S}}, q=1, \ldots, Q, \\
z_{i q}^{\mathrm{E}} \leq z_{i, q-1}^{\mathrm{S}}, \quad i \in \mathcal{I}_{q}^{\mathrm{B}}, q=1, \ldots, Q, \\
\sum_{i \in \mathcal{I}} p_{i} z_{i q}^{\mathrm{E}}+\sum_{q^{\prime}=1}^{q} u_{q^{\prime}}^{\mathrm{idle}} \leq t_{q}, \quad q=1, \ldots, Q, \\
\sum_{i \in \mathcal{I}} p_{i} z_{i q}^{\mathrm{S}}+\sum_{q^{\prime}=1}^{q} u_{q^{\prime}}^{\mathrm{idle}} \geq t_{q}, \quad q=1, \ldots, Q, \\
u_{q}^{\mathrm{idle}} \leq\left(t_{q}-t_{q^{-1}}\right)\left(1-\sum_{i \in \mathcal{I}}\left(z_{i q}^{\mathrm{S}}-z_{i, q+1}^{\mathrm{E}}\right)\right), \quad q=1, \ldots, Q, \\
\sum_{i \in \mathcal{I}}\left(z_{i q}^{\mathrm{S}}-z_{i q}^{\mathrm{E}}\right) \leq 1, \quad q=1, \ldots, Q, \\
\sum_{i \in \mathcal{I}_{q}^{\mathrm{B}}}^{\mathrm{B}}\left(z_{i, q-1}^{\mathrm{S}}-z_{i, q}^{\mathrm{E}}\right)+z_{i^{\prime} q}^{\mathrm{E}}-z_{i^{\prime}, q-1}^{\mathrm{S}} \leq 1, \quad i^{\prime} \in \mathcal{I}_{q}^{\mathrm{S}}, q=1, \ldots, Q, \\
z_{i q}^{\mathrm{S}}=0, \quad q=1, \ldots, Q, i \in \mathcal{I}: t_{q} \leq r_{i}, \\
z_{i q}^{\mathrm{E}}=1, \quad q=1, \ldots, Q, i \in \mathcal{I}: t_{q} \geq d_{i},
\end{gathered}
$$

we can find a feasible schedule. Constraints (3.6)-(3.7) ensure consistent definitions of the binary variables. Constraints (3.8) make sure that if a task is completed in some interval, it should be started in this interval or earlier. Constraints (3.9) make sure that, if a task does not fit inside an interval, it cannot start before this interval and be completed in a later interval. Constraints (3.10) ensure that tasks that are larger than an interval cannot start 
and be completed in that particular interval. Constraints (3.11)-(3.12) ensure that the sum of processing times of tasks scheduled up to the start of an interval plus the idle time of the intervals are consistent with the time spent. Constraints (3.13) put the idle times of an interval to 0 if a task starts before the interval and is completed after the interval. Constraints (3.14) state that there is at most one task that is started before the end of an interval and finished after it. Constraints (3.15) eliminate for each interval, overlap between tasks that are too large to be fully processed within the interval and tasks that are fully processed within the interval. Constraints (3.16)-(3.17) fix appropriate binary variables to account for release times and deadlines.

The idea of dividing the scheduling horizon into intervals and assigning tasks to start within the intervals is also the main idea behind the LBBD schemes introduced in [9]. In [9], two single machine scheduling problems are studied. In first problem, the scheduling horizon is divided into disjoint segments. Each task in the problem must be fully performed within one of the segments. This can be considered a special case of the single machine scheduling problem with multiple time-windows and setup times described later in this chapter. The idea behind the LBBD scheme for this problem is to assign an interval to each task in a master problem and evaluate an assignment of intervals to tasks in a subproblem. In the second problem, there are no disjoint segments. Instead, a division of the scheduling horizon is done a priori. In the LBBD scheme for this problem, each task is assigned to start within one interval in a master problem and this assignment is evaluated in a subproblem. For both problems, the authors evaluate a feasibility version, the minimisation of makespan, and the minimisation of tardiness.

\section{Bucket-indexed formulation}

The bucket-indexed formulation was introduced in [7]. In this formulation, the scheduling horizon is divided into buckets of equal length. However, the bucket length $p^{\mathrm{B}}$ is selected to be less than or equal to the shortest processing time of all tasks and larger then or equal to the unit size time step, hence $1 \leq p^{\mathrm{B}} \leq \min _{i \in \mathcal{I}} p_{i}$. The reasons for this are to make sure that only a single task can start in a bucket and that each task must span at least two buckets. The bucket-indexed formulation can be seen as an extension of the time-indexed formulation, since the special case of the bucket formulation where the bucket length is equal to the unit-size is the time-indexed formulation.

To construct the model, we introduce some notation. Let $B$ denote the number of buckets for a division of the scheduling horizon with bucket length $p^{\mathrm{B}}$. Specifcally, a bucket $b=1, \ldots, B$, corresponds to the right half-open real interval $\left[(b-1) p^{\mathrm{B}}, b p^{\mathrm{B}}\right)$ Additionally, assume that the tasks in the set $\mathcal{I}$ are ordered according to non-decreasing processing times. For each task $i \in \mathcal{I}$, let $l_{i}^{\mathrm{B}}=\left\lfloor p_{i} / p^{\mathrm{B}}\right\rfloor$ denote the number of buckets it must at least span and let $\pi_{i}=l_{i}^{\mathrm{B}}-p_{i} / p^{\mathrm{B}}$ denote the fraction of a bucket length it must be span besides 
the complete bucket lenghts. Note that $p_{i}=\left(l_{i}^{\mathrm{B}}-\pi_{i}\right) p^{\mathrm{B}}$, for task $i \in \mathcal{I}$. For each task $i \in \mathcal{I}$, let $R_{i}=\left\lfloor r_{i} / p^{\mathrm{B}}\right\rfloor+1$ denote the bucket its release time occurs in and let $\rho_{i}^{\mathrm{r}}=R_{i}-r_{j} / p^{\mathrm{B}}$ denote the offset inside the bucket the release time occurs. For each task $i \in \mathcal{I}$, also let $D_{i}=\left\lfloor d_{i} / p^{\mathrm{B}}\right\rfloor+1$ denote the bucket its deadline occurs in and let $\rho_{i}^{\mathrm{d}}=D_{i}-d_{j} / p^{\mathrm{B}}$ denote the offset inside the bucket the deadline occurs.

For each task $i \in \mathcal{I}, k \in\{0,1\}$ and bucket $b=1, \ldots, B$, introduce the binary variable

$$
z_{i b k}= \begin{cases}1, & \text { if task } i \text { starts in bucket } b \text { and finishes in bucket } b+l_{i}^{\mathrm{B}}+k, \\ 0, & \text { otherwise. }\end{cases}
$$

A task $i \in \mathcal{I}$ is said to span $m$ buckets if it starts in bucket $b$ and is completed in bucket $b+m-1$. Also, for each task $i \in \mathcal{I}, k \in\{0,1\}$, and bucket $b=1, \ldots, B$, introduce the continuous variables

$$
u_{i b k}^{\mathrm{S}}=\left\{\begin{array}{l}
\text { fraction of task } i \text { spent in bucket } b \text { if task } i \text { starts in bucket } b \\
\text { and spans } l_{i}^{\mathrm{B}}+k \text { buckets, } 0 \text { otherwise, }
\end{array}\right.
$$

and

$u_{i b k}^{\mathrm{C}}=\left\{\begin{array}{l}\text { fraction of task } i \text { spent in bucket } b \text { if task } i \text { is completed in bucket } b \\ \text { and spanned } l_{i}^{\mathrm{B}}+k \text { buckets, } 0 \text { otherwise. }\end{array}\right.$

The bucket-indexed formulation is

$$
\begin{gathered}
\sum_{k \in\{0,1\}} \sum_{b=1}^{B} z_{i b k}=1, \quad i \in \mathcal{I}, \\
\sum_{i \in \mathcal{I}} \sum_{k \in\{0,1\}} \sum_{b^{\prime}=b-l_{i}^{\mathrm{B}}-k+2}^{b} z_{i b^{\prime} k} \leq 1, \quad b=1, \ldots, B, \\
\sum_{i \in \mathcal{I}} \sum_{k \in\{0,1\}}\left(u_{i b k}^{\mathrm{S}}+u_{i b k}^{\mathrm{C}}+\sum_{b^{\prime}=b-l_{i}^{\mathrm{B}}-k+2}^{b-1} z_{i b^{\prime} k}\right) \leq 1, \quad b=1, \ldots, B, \\
u_{i b k}^{\mathrm{S}}+u_{i, b+l_{i}^{\mathrm{B}}+k-1, k}^{\mathrm{C}}=\left(2-k-\pi_{i}\right) z_{i b k}, \quad i \in \mathcal{I}, \quad k \in\{0,1\}, b=1, \ldots, B, \\
\left((1-k)\left(1-\pi_{i}\right)+1 / p^{\mathrm{B}}\right) z_{i b k} \leq u_{i b k}^{\mathrm{S}}, \quad i \in \mathcal{I}, \quad k \in\{0,1\}, b=1, \ldots, B, \\
u_{i b k}^{\mathrm{S}} \leq\left(1-k \pi_{i}\right) z_{i b k}, \quad i \in \mathcal{I}, k \in\{0,1\}, b=1, \ldots, B, \\
(1-k)\left(1-\pi_{i}\right) z_{i b k} \leq u_{i, b+l_{i}^{\mathrm{B}}+k-1, k}^{\mathrm{C}}, \quad i \in \mathcal{I}, \quad k \in\{0,1\}, b=1, \ldots, B, \\
u_{i, b+l_{i}^{\mathrm{B}}+k-1, k}^{\mathrm{C}} \leq\left(1-k \pi_{i}-1 / p^{\mathrm{B}}\right) z_{i b k}, \quad i \in \mathcal{I}, \quad k \in\{0,1\}, b=1, \ldots, B, \\
u_{i R_{i} 0}^{\mathrm{S}} \leq \rho_{i}^{\mathrm{r}} z_{i R_{i} 0}, \quad i \in \mathcal{I}: 1-\pi_{i}<\rho_{i}^{\mathrm{r}}<1, \\
u_{i R_{i} 1}^{\mathrm{S}} \leq \rho_{i}^{\mathrm{r}} z_{i R_{i} 1}, \quad i \in \mathcal{I}: \rho_{i}^{\mathrm{r}}<1-\pi_{i}, \\
\rho_{i}^{\mathrm{d}} z_{i D_{i} 0} \leq u_{i D_{i} 0}^{\mathrm{S}}, \quad i \in \mathcal{I}: 1-\pi_{i}<\rho_{i}^{\mathrm{d}}<1, \\
\rho_{i}^{\mathrm{d}} z_{i D_{i} 1} \leq u_{i D_{i} 1}^{\mathrm{S}}, \quad i \in \mathcal{I}: \rho_{i}^{\mathrm{d}}<1-\pi_{i} .
\end{gathered}
$$


Constraints (3.18) make sure that each task starts in a bucket. Constraints (3.19)-(3.20) ensure that only one task can be performed at a given time. Constraints (3.21)-(3.25) make sure that the continuous and binary variable get the correct definitions. Constraints (3.26)-(3.27) ensure that release times are respected. Constraints (3.28)-(3.29) ensure that deadlines are respected.

The bucket-indexed formulation is theoretically at least as strong as the time-indexed formulation, which is proven in [7]. In [39], to solve a resourceconstrained project scheduling problem, a bucket-indexed approach is implemented in an iterative framework, that, if necessary, divides the scheduling horizon into smaller and smaller pieces.

\subsubsection{Order-oriented formulations}

The order-oriented formulations are centred around assuring that pairs of tasks do not overlap. We will introduce two formulations that both use binary variables to indicate in which order pairs of tasks occur and continuous variables that represent the start times of tasks. By connecting the ordervariable for each pair of tasks to their start time variables via constraints, each pair of tasks can be ensured not to overlap. How this connection is done is the main difference between the two formulations.

Both order-oriented formulations use the following variables. Introduce, for each pair of tasks $i, i^{\prime} \in \mathcal{I}: i \neq i^{\prime}$, the binary variable

$$
y_{i i^{\prime}}= \begin{cases}1, & \text { if task } i \text { is performed before task } i^{\prime}, \\ 0, & \text { otherwise. }\end{cases}
$$

For each task $i \in \mathcal{I}$, introduce the continuous variable

$$
s_{i}=\text { start time of task } i \text {. }
$$

\section{Disjunctive formulation}

The disjunctive formulation can, with the variables introduced above, be formulated as

$$
\begin{gathered}
s_{i^{\prime}} \geq s_{i}+p_{i}-M\left(1-y_{i i^{\prime}}\right), \quad i, i^{\prime} \in \mathcal{I}: i \neq i^{\prime}, \\
y_{i i^{\prime}}+y_{i^{\prime} i}=1, \quad i, i^{\prime} \in \mathcal{I}: i<i^{\prime}, \\
r_{i} \leq s_{i} \leq d_{i}-p_{i}, \quad i \in \mathcal{I},
\end{gathered}
$$

where the constant $M$ is sufficiently large. Constraints (3.32)-(3.33) ensure that no two tasks overlap. Constraints (3.34) make sure that each task respects its release time and deadline. Note that constraints (3.32) is of big-M type and will typically yield a weak Linear Programming (LP) relaxation. 
The order-oriented decision variable for a scheduling problem was introduced in [31] in the context of job-shop scheduling. In the same year, a variant of defining an order-oriented variable was also studied in [32] for the travelling salesman problem. In this setting, each binary variable denoted if a task was the immediate predecessor to another task in a schedule or not.

The polyhedral studies of disjunctive formulation for the single machine scheduling problem were started in [4], who studied the polyhedra in the context of job shop scheduling for a wide range of constraints. An extended description of the polyhedra of the disjunctive formulation was given a few years later in [35], who utilised the results in a cutting plane procedure.

\section{Linear-ordering formulation}

The linear-ordering formulation is a variant of the disjunctive formulation where the order-oriented decision variables are connected with the triangle inequality instead of big-M constraints. In order to get a well-defined schedule, an objective function to minimise the sum of the starting times is used. Additionally, assume that the tasks in the set $\mathcal{I}$ are ordered with respect to non-increasing order of their release times and set $y_{i i}=1$ for each task $i \in \mathcal{I}$.

By solving

$$
\begin{array}{ll}
\min & \sum_{i \in \mathcal{I}} s_{i}, \\
\text { s.t. } & y_{i j}+y_{j i}=1, \quad i, i^{\prime} \in \mathcal{I}, \quad i<i^{\prime}, \\
& y_{i i^{\prime}}+y_{i^{\prime} i^{\prime \prime}}+y_{i^{\prime \prime} i} \leq 2, \quad i, i^{\prime}, i^{\prime \prime} \in \mathcal{I}: i \neq i^{\prime}, i^{\prime} \neq i^{\prime \prime}, i \neq i^{\prime \prime}, \\
& s_{i^{\prime}} \geq r_{i} y_{i i^{\prime}}+\sum_{\substack{i^{\prime \prime} \in \mathcal{I}: \\
i^{\prime \prime}<i, i^{\prime \prime} \neq i^{\prime}}} p_{i^{\prime \prime}}\left(y_{i i^{\prime \prime}}+y_{i^{\prime \prime} i^{\prime}}-1\right)+\sum_{\substack{i^{\prime \prime} \in \mathcal{I}: \\
i^{\prime \prime} \geq i, i^{\prime \prime} \neq i^{\prime}}} p_{i^{\prime \prime}} y_{i^{\prime \prime} i^{\prime}}, \quad i, i^{\prime} \in \mathcal{I}, \\
& s_{i} \leq d_{i}-p_{i}, \quad i \in \mathcal{I},
\end{array}
$$

we can find a feasible schedule for the single machine scheduling problem with release times and deadlines if one exists. The objective function is to minimise the sum of the start times. Constraints (3.36)-(3.37) ensure a valid sequence. Constraints (3.38) force each task to respect its release time while making sure that tasks does not start before preceding tasks are finished. Constraints (3.39) force each task to finish before its deadline. Note that any feasible order given by the binary decisions $y_{i j}$ will have at least one corresponding feasible schedule where all tasks are scheduled as early as possible with respect to their order.

In [11], constraints (3.37) were first used as an extension to the disjunctive formulation when studying theoretical properties of formulations. However, the linear ordering formulation as shown above was introduced in [33], along with some valid inequalities. 


\subsubsection{Position-oriented formulation}

The position-oriented formulation is organised around the notion that a schedule can be represented by the position each task occupies. The formulation includes binary decision variables that indicate if a task is performed at a specific position and a continuous variable for each position that represent the start time of the task processed at that position.

Let $P=|\mathcal{I}|$ denote the number of positions. For task $i \in \mathcal{I}$ and position $p=1, \ldots, P$, introduce the binary variable

$$
z_{i p}= \begin{cases}1, & \text { if task } i \text { is performed in position } p \\ 0, & \text { otherwise }\end{cases}
$$

For position $p=1, \ldots, P$, introduce the continuous variable

$$
\mu_{p}=\text { start time of the task that is performed in position } p \text {. }
$$

A solution to

$$
\begin{gathered}
\sum_{p=1}^{P} z_{i p}=1, \quad i \in \mathcal{I}, \\
\sum_{i \in \mathcal{I}} z_{i p}=1, \quad p=1, \ldots, P, \\
\mu_{p}+\sum_{i \in \mathcal{I}} z_{i p} p_{i} \leq \mu_{p+1}, \quad p=1, \ldots, P-1, \\
\sum_{i \in \mathcal{I}} z_{i p} r_{i} \leq \mu_{p} \leq \sum_{i \in \mathcal{I}} z_{i p}\left(d_{i}-p_{i}\right), \quad p=1, \ldots, P,
\end{gathered}
$$

will be a feasible schedule to the single machine scheduling problem with release times and deadlines. Constraints (3.42) assign each task a position. Constraints (3.43) assign each position a task. Constraints (3.44) ensure that two tasks at adjacent positions do not overlap. Constraints (3.45) make sure that each task respects its release time and deadline. Since there is no explicit connection between a task and its starting time, this formulation is a little less flexible dealing with time-related constraints, e.g., multiple time-windows, minimal time lags, and maximum time lags, without introducing constructions like big-M constraints.

The notion of using a position-oriented variable in order to formulate an MIP for a scheduling problem was first introduced in [44] in the context of machine scheduling. In [29], the polyhedra of the position-oriented formulation was studied and valid inequalities was introduced. In recent years, the authors of [28] introduced additional valid inequalities for the position-oriented formulation based on the shortest processing time list-scheduling rule. In the same year, [10] used the position-oriented formulation with some valid inequalities to explore neighbourhoods in a matheuristic. 


\subsubsection{Comparisons of mixed-integer programming formulations}

The MIP formulations introduced in the previous sections have been compared in different settings in the literature. There have been a few theoretical comparisons and primarily computational comparisons. This section attempts to summarise these results.

In [11], the authors studied a series of LP relaxations originating from the disjunctive and time-oriented formulations. The authors showed that the LP relaxation of the time-oriented formulation dominates the LP relaxation of the disjunctive formulation for the problem of minimising the weighted sum of start times for the single machine problem with release times $\left(1\left|r_{j}\right| \sum w_{j} S_{j}\right)$. A similar property is shown in [7], where the LP relaxation of the bucketindexed formulation is shown to be at least as strong as the time-indexed formulation.

Four MIP formulations, the disjunctive, the time indexed, the linear ordering, and the position oriented, were compared in [27]. Computational tests were conducted for the single machine scheduling problem when minimising the weighted completion time, the maximum lateness, the number of tardy jobs, and the total weighted tardiness. These comparisons were made both with and without release times, hence some of the problems are NP hard and some can be solved in polynomial time. Their results indicated that the LP relaxations of the disjunctive formulation and the position-oriented formulation were easier to solve than the LP relaxations of the time-indexed formulation and the linear ordering formulation, but they achieved weaker bounds. Among the four formulations, the authors observed that the position-oriented and the disjunctive formulation found feasible solutions first even though the obtained solutions were not always optimal.

A year later, computational comparisons between six MIP formulations were performed in [3]. Four of the formulations were the disjunctive, the time indexed, the position oriented, and the linear-ordering. The additional two formulations were a hybrid of disjunctive and position-oriented formulations, and a travelling salesman problem inspired formulation. The comparison was made for the single machine scheduling problem without release times while attempting to minimise total tardiness. Their results indicated that the position-oriented formulations performed best in reducing the total tardiness while the hybrid version and the travelling salesman problem formulation performed worst.

When introducing the interval-indexed formulation in [5], the authors made a computational comparison with other formulations. For the single machine scheduling problem with a generic objective function and release times $\left(1\left|r_{j}\right| \sum \alpha_{j} E_{j}+\beta_{j} T_{j}\right.$ ), the authors compared the interval-indexed formulation with the disjunctive formulation and the time-indexed formulation. In this comparison, the time-indexed formulation solved most instances 
to optimality followed by the interval-indexed formulation, and last was the disjunctive formulation. Worth noting is that the disjunctive formulation processed nodes in the MIP solver much faster than the time-indexed formulation on these instances. The authors also made a comparison on a problem without release times $\left(1|-| \sum w_{j} T_{j}\right)$. In this setting, they compared the intervalindexed formulation, the time-oriented formulation, and the linear-ordering formulation. In this comparison, the interval-indexed formulation performed best with respect to the relative MIP-gap, but when comparing the number of instances solved to optimality, none of the formulations performed better than the others.

\subsection{Constraint programming models}

A different paradigm to solve scheduling problem is CP. For an introduction to CP, see [41]. Simply speaking, CP uses global constraints such as Disjunctive, Cumulative, and AllDifferent to describe common pattern of constraints, which together with the possibility to uses lower-level expressions such as binary variables, integer variables, interval variables, and inequality constraints makes its modelling language rich. The global constraints make it possible for $\mathrm{CP}$ solvers to implement specific strategies within the solvers to handle the constraints, which can benefit the efficiency of the search. CP solvers are in general exact but the difference in computational performance between solvers can be large and different solvers can perform better on (or be limited to) certain variables and global constraints. One area of great success for $\mathrm{CP}$ is to model and solve scheduling problems, using global constraints such as Disjunctive and Cumulative. The single machine scheduling problem with release times and deadlines can be compactly expressed using the DisJunCTIVE constraint and such a model is presented in the following subsection.

\subsubsection{Disjunctive-interval formulation}

The disjunctive-interval formulation uses interval variables and the global CP constraint DisJunCtive to ensure that no tasks overlap. An interval variable is a special type of decision variable that represents an interval of time whose position in time is unknown and needs to be decided. An interval variable $s_{i}$ is associated with a release time $r_{i} \in \mathbb{Z}$ and a deadline $d_{i} \in \mathbb{Z}$, that define its domain $\left[r_{i}, d_{i}\right]$. The decisions of an interval variable $s_{i}$ are normally to select a start time $\operatorname{start}\left(s_{i}\right)$ and an end time end $\left(s_{i}\right)$ such that $r_{i} \leq \operatorname{start}\left(s_{i}\right) \leq$ end $\left(s_{i}\right) \leq d_{i}$. The processing time or length of the interval variable $s_{i}$ is then $\operatorname{proc}\left(s_{i}\right)=\operatorname{end}\left(s_{i}\right)-\operatorname{start}\left(s_{i}\right)$. However, in our modelling, we only use interval variables with a fixed length and in the formulation, the processing times are added as input to the DisJunCTIVE constraint where they are used. 
For each task $i, i \in \mathcal{I}$, introduce the interval variable

$$
s_{i}=\text { start time of task } i \text {. }
$$

To ensure that the start times of tasks do not overlap, the global constraint Disjunctive is used. The global constraint DisJunctive is defined for an array of interval variables $\left(s_{1}, \ldots, s_{n}\right)$ and an array of processing times $\left(p_{1}, \ldots, p_{n}\right)$. An interval variable $s_{i}, i=1, \ldots, n$, is assumed to be active in the constraint if $\operatorname{start}\left(s_{i}\right) \leq t<\operatorname{start}\left(s_{i}\right)+p_{i}$. The constraint stipulates that for each time point $t \in \mathbb{Z}$ at most one interval variable can be active.

Utilising this, the single machine scheduling problem with release times and deadlines can be formulated as

$$
\begin{gathered}
\operatorname{DisjunCtive}\left(\left(s_{i} \mid i \in \mathcal{I}\right),\left(p_{i} \mid i \in \mathcal{I}\right)\right), \\
r_{i} \leq s_{i} \leq d_{i}-p_{i}, \quad i \in \mathcal{I},
\end{gathered}
$$

in what we refer to as the disjunctive-interval formulation. Constraint (3.47) ensures that no tasks overlap. Constraints (3.48) ensure that the release time and deadline of each task are obeyed.

\subsection{Two decomposition schemes for a single machine scheduling problem}

This section aims at describing the connection between the constraint generation procedure from Paper A and Paper B, the LBBD schemes in Paper C and Paper D, and the MIP and CP formulations for the single machine scheduling problem with release times and deadlines presented in this chapter. To do so, we present two decomposition schemes in the context of a single machine scheduling problem. To intention is to illustrate the different feedback systems and the subproblems used in the decomposition schemes without including the constraints that are not central to the idea behind the decompositions. However, both these decomposition schemes are in their original versions applied to problems where each task must be placed within one of its time-windows (or sub-intervals) which is reflected in their master problem variables. So instead of describing the decompositions for the single machine scheduling problem with release times and deadlines, which does not include multiple time-windows, the decompositions are described for the single machine scheduling problem with sequence-dependent setup times and multiple time-windows studied in Paper D. 


\subsubsection{A single machine scheduling problem with sequence-dependent setup times and multiple time-windows}

The single machine scheduling problem with sequence-dependent setup times and multiple time-windows was introduced in [19] and is relevant for the avionics scheduling problem design case. The problem can be seen as an extension of the single machine scheduling problem with release times and deadlines introduced in Section 3.1. The extension is that it includes multiple time-windows instead of one, sequence-dependent setup times between tasks, and instead of a feasibility problem, the purpose is to maximise the weighted sum of the tasks that can be scheduled.

Let $\mathcal{I}$ denote the set of tasks that can be scheduled. A prize $q_{i}$ is collected if task $i \in \mathcal{I}$ is scheduled in the interval between the release time $r_{i q}$ and deadline $d_{i q}$ of one of its time-windows $q \in \mathcal{Q}_{i}$. Each scheduled task $i \in \mathcal{I}$ must be given exclusive uninterrupted access to the machine for the duration of its processing time $p_{i}$. If task $i$ is performed before task $j$, there must be a minimum setup time $s_{i j}$ between the end of task $i$ and the start of task $j$, $i, j \in \mathcal{I}: i \neq j$. The objective can be seen as a variant of the weighted tardy jobs objective where instead of penalising if a task is not scheduled, a prize is collected if a task is scheduled.

\subsubsection{A common decomposition idea}

The idea behind both decomposition schemes is to assign time-windows to tasks in a master problem. The tasks that have been assigned a time-window in the master problem are then included in a subproblem, where they must be performed within their assigned time-window. If the tasks in the subproblem cannot be scheduled, constraints are generated. The differences between the decomposition schemes are in their feedback mechanism and in their subproblem formulation. However, the schemes share some similar concepts, such as the main decision variables, the structure of the master problem without the feedback, and how a solution to the master problem is used to create the subproblem. These concepts are explained in this section.

The purpose of the master problem is to create an assignment of timewindows to tasks. For time-window $q \in \mathcal{Q}_{i}$ and task $i \in \mathcal{I}$, introduce the binary variable

$$
x_{i q}= \begin{cases}1, & \text { if task } i \text { is assigned to time-window } q, \\ 0, & \text { otherwise. }\end{cases}
$$


The master problem of both decomposition schemes can then be formulated as

$$
\begin{aligned}
{\left[\mathrm{MP}^{k}\right] \max } & \sum_{i \in \mathcal{I}} \sum_{q \in \mathcal{Q}_{i}} q_{i} x_{i q}, \\
\text { s.t. } & \sum_{q \in \mathcal{Q}_{i}} x_{i q} \leq 1, \quad i \in \mathcal{I}, \\
& {[\text { Segment relaxtion }], } \\
& {\left[\text { Feedback }{ }^{k}\right] }
\end{aligned}
$$

for iteration $k$ of the scheme. The objective function (3.49) maximises the weighted sum of the tasks that are scheduled. Constraints (3.50) ensure that at most one time-window can be assigned to each task. To strengthen the master problem, we use the [Segment relaxation] described in Paper D which ensures that the capacity to perform tasks for certain parts of the timeline is not exceeded. The [Segment relaxation] is formulated as

$$
\sum_{i \in \mathcal{I}} \sum_{q \in \mathcal{Q}_{i}\left(t_{1}, t_{2}\right)} p_{i} x_{i q} \leq t_{2}-t_{1}, \quad\left(t_{1}, t_{2}\right) \in T
$$

where $T=\left\{\left(r_{i q}, d_{i^{\prime} q^{\prime}}\right):\left(q, q^{\prime}\right) \in Q_{i} \times Q_{i^{\prime}},\left(i, i^{\prime}\right) \in I \times I, d_{i^{\prime} q^{\prime}}>r_{i q}\right\}$ is the set of release time and deadline pairs for which the segment relaxation is used. Further, the set $\mathcal{Q}_{i}\left(t_{1}, t_{2}\right)=\left\{q \in \mathcal{Q}_{i}: t_{1} \leq r_{i q}, d_{i q} \leq t_{2}\right\}$ gives the time-windows for task $i \in \mathcal{I}$ that starts after $t_{1}$ and ends before $t_{2}$. The [Feedback ${ }^{k}$ ] component ensures that the method converges and is uniquely implemented for each decomposition scheme below. Note that the idea of assigning time-windows to tasks is somewhat similar to the idea behind two of the main decision variables in the interval-indexed formulation presented earlier in this chapter.

A solution to a master problem $\mathrm{MP}^{k}$ is used to construct the subproblem, that is a restriction of the original problem. The subproblem and the feedback are different in the two decomposition schemes, but the following notation is used in both. Let the subset of tasks $\overline{\mathcal{I}}^{k} \subseteq \mathcal{I}$ be the tasks assigned a time-window in the master problem of iteration $k$ and let $\bar{q}_{i}^{k}$ be the assigned time-window of each task $i \in \overline{\mathcal{I}}^{k}$.

\subsubsection{A constraint generation scheme}

In this section, we present a constraint generation scheme for the single machine scheduling problem with multiple time-windows and sequencedependent setup time. This scheme is inspired by the constraint generation procedure for the avionics scheduling problem from Papers A and B. Even though the problems are quite different, a key component of the constraint generation procedure for the avionics scheduling problem is to handle the challenge of scheduling tasks in two steps. This section tries to highlight this idea by applying it in a cleaner context. 
In this constraint generation scheme for the single machine scheduling problem with multiple time-windows and sequence-dependent setup time, the subproblem is constructed from the tasks that were assigned time-windows in a master problem solution. In the subproblem, the scheduling constraints of the tasks are soft, and the objective is to minimise the number of tasks that cannot be scheduled. If a solution to the subproblem is found where all tasks in the subproblem are scheduled, the original problem is solved. If no solution without violation is found, scheduling constraints are added to the master problem and the subproblem in subsequent iterations. A positive aspect of this scheme is that the subproblem does not have to be solved to optimality and can be exited after a given time limit. If no tasks can be scheduled in the original problem, the master problem of the scheme will converge to a complete formulation of the original problem, proving infeasibility. The subproblem can be categorised as $\left(1\left|r_{i}\right| \sum U_{i}\right)$.

Formally, the subproblem in iteration $k$ search for the largest subset of tasks that can be scheduled within their assigned time-windows without overlapping each other. If a task is not included in this subset, it is referred to as being tardy. The main decision variables are to decide start times for the tasks and if they are tardy or not. In iteration $k$, introduce for each task $i \in \overline{\mathcal{I}}_{k}$, the binary variable

$$
\beta_{i}= \begin{cases}1, & \text { if task } i \text { is tardy } \\ 0, & \text { otherwise }\end{cases}
$$

and the continuous variable

$$
y_{i}=\text { start time of task } i \text {. }
$$

Also let for $i, i^{\prime} \in \overline{\mathcal{I}}^{k}: i \neq i^{\prime}$, in iteration $k$, the binary variable $y_{i i^{\prime}}^{\mathrm{s}}$ indicate if task $i$ is scheduled before task $i^{\prime}$ or not.

The subproblem is then formulated as

$$
\begin{aligned}
& {\left[\mathrm{SP}-\mathrm{CG}{ }^{k}\right] \min } \sum_{i \in \overline{\mathcal{I}}^{k}} \beta_{i}, \\
& \text { s.t. } 1-\beta_{i}-\beta_{i^{\prime}} \leq y_{i i^{\prime}}^{\mathrm{s}}+y_{i^{\prime} i}^{\mathrm{s}} \leq 1, \quad i, i^{\prime} \in \overline{\mathcal{I}}^{k}: i \neq i^{\prime}, \\
& y_{i}+p_{i}+s_{i i^{\prime}}-M\left(1-y_{i i^{\prime}}^{\mathrm{s}}\right) \leq x_{i^{\prime}}, \quad i, i^{\prime} \in \overline{\mathcal{I}}^{k}: i \neq i^{\prime}, \\
& r_{i \bar{q}_{i}^{k}} \leq y_{i} \leq d_{i \bar{q}_{i}^{k}}-p_{i}, \quad i \in \overline{\mathcal{I}}^{k}, \\
& {\left[\text { Feedback-SP-CG }{ }^{k}\right], }
\end{aligned}
$$

where the constant $M$ is selected to be sufficiently large. The objective function (3.54) minimises the number of tardy tasks. Constraints (3.55) ensure that if two tasks are not tardy, one must be performed before the other. Constraints (3.56) ensure that if two tasks are not tardy, they cannot overlap. Constraints (3.57) make sure that each task is performed within its 
time-window. The $\left[\right.$ Feedback-SP $\left.{ }^{k}\right]$ component ensures that the constraints that were generated in previous iterations are not generated again. The subproblem formulation can be seen as a variant of the MIP-based disjunctive formulation.

If no solution to the subproblem is found where there are no tardy tasks, feedback is added to the master problem and the subproblem using the tasks that were tardy. Let $\hat{\mathcal{I}}^{k}=\left\{i \in \mathcal{I}^{k}: \beta_{i}=1\right\}$ denote the set of tardy tasks in the best solution to subproblem SP-CG ${ }^{k}$ in iteration $k$. To help with the feedback in the master problem, introduce the following variables in the master problem [Feedback] component. Introduce for task $i \in \mathcal{I}$, a continuous variable $y_{i}$ that represents the start time of task $i$. Also let, for $i, i^{\prime} \in \mathcal{I}: i \neq i^{\prime}$, the binary variable $y_{i i^{\prime}}^{\mathrm{s}}$ indicate if task $i$ is scheduled before task $i^{\prime}$ or not.

The master problem feedback component in iteration $k$ can then be formulated as

$$
\begin{gathered}
{\left[\text { Feedback-CG }{ }^{k}\right] y_{i i^{\prime}}^{\mathrm{s}}+y_{i^{\prime} i}^{\mathrm{s}}=1, \quad\left(i, i^{\prime}\right) \in \hat{\mathcal{I}}^{k},} \\
y_{i}+p_{i}+s_{i i^{\prime}}-M\left(1-y_{i i^{\prime}}^{\mathrm{s}}\right) \leq y_{i^{\prime}}, \quad i, i^{\prime} \in \overline{\mathcal{I}}^{k}, i \neq i^{\prime}, \\
\\
\sum_{q \in \mathcal{Q}_{i}} r_{i q} x_{i q} \leq y_{i} \leq \hat{M}-\sum_{q \in \mathcal{Q}_{i}}\left(\hat{M}-d_{i q}\right) x_{i q}-p_{i}, \quad i \in \mathcal{I},
\end{gathered}
$$

where $\hat{\mathcal{I I}}^{k}=\left\{\left(i, i^{\prime}\right) \in \mathcal{I} \times \mathcal{I}: i, i^{\prime} \in \cup_{k^{\prime}=1, \ldots, k} \overline{\mathcal{I}}^{k^{\prime}}, i<i^{\prime}\right\}, \hat{\mathcal{I} \mathcal{I}^{1}}=\varnothing$, and the constants $M$ and $\hat{M}$ are sufficiently large. Constraints (3.59) ensure that for each pair of tardy tasks that has been generated, one must be placed before the other. Constraints (3.60) ensure that no overlaps occur when one task is performed before the other. Constraints (3.61) make sure that tasks are given start times within their assigned time-window. The key is that overlap constraints are only enforced for tasks that have been tardy in the subproblem.

To avoid generating the same tardy tasks in subsequent subproblems, the constraints

$$
\left[\text { Feedback-SP-CG }{ }^{k}\right] \beta_{i}=1, \quad i \in \hat{\mathcal{I}}^{k^{\prime}}: i \neq i^{\prime}, k^{\prime}=1, \ldots, k-1,
$$

are included in subproblems.

This scheme may see somewhat naive when applied to this type of problem, but there are some characteristics in the avionics scheduling problem for which it was introduced, that makes it suitable. The avionics scheduling problem includes more constraints than the single machine scheduling problem with multiple time-windows and sequence-dependent setup times. It includes, e.g., precedence constraints and scheduling of additional machines (modules). By assigning time-windows to tasks in the avionics scheduling problem, certain tasks will never overlap. This ensures that some tasks will not overlap in the subproblem, and the scheduling becomes somewhat of a local issue. This is exploited both in how infeasibilities are measured but also in the construction of feedback. Instead of minimising the number of tardy tasks, the objective in 
Paper B is about minimising the number of sequences (combinations of tasks that can overlap) that includes no overlaps between tasks. Also, instead of generating tardy tasks, the sequences of tasks that are generated are forced not to overlap in the subsequent master problems and subproblems.

\subsubsection{A logic-based Benders decomposition scheme}

In this section, we present the LBBD scheme for the single machine scheduling problem with multiple time-windows and sequence-dependent setup times introduced in Paper D. It is a generalisation of the model used in [9] for a single machine scheduling problem with a segmented timeline since it allows for unique time-windows for each task and because it includes sequencedependent setup times. The idea of the decomposition is similar to the one used for the avionics scheduling problem in Paper C.

In this LBBD scheme, the subproblem is constructed from the tasks that were assigned time-windows in a master problem solution. The subproblem uses the CP-based disjunctive-interval formulation to check if a feasible schedule can be found with the assigned time-windows. If there exists a feasible schedule, the original problem has been solved. If there exists no feasible schedule, a feasibility cut is added to the master problem. The subproblem can be categorised as $\left(1\left|r_{i}, \bar{d}_{i}\right|-\right)$.

In the subproblem, introduce for each task $i \in \mathcal{I}$, the interval variable

$$
y_{i}=\text { start time of task } i \text {. }
$$

The subproblem can then be formulated as

$\left[\mathrm{SP}-\mathrm{LD}^{k}\right] \min 0$,

$$
\begin{aligned}
& \text { s.t. Disjunctive }\left(\left(y_{i} \mid i \in \overline{\mathcal{I}}^{k}\right),\left(p_{i} \mid i \in \overline{\mathcal{I}}^{k}\right),\left(s_{i j} \mid i, j \in \overline{\mathcal{I}}^{k}\right)\right) \text {, } \\
& r_{i \bar{q}_{i}^{k}} \leq y_{i} \leq d_{i \bar{q}_{i}^{k}}-p_{i}, \quad i \in \overline{\mathcal{I}}^{k} .
\end{aligned}
$$

Constraint (3.63) ensures that no interval variables overlap while obeying the sequence-dependent setup times using a variant of the DisJUNCTIVE constraint. Constraints (3.64) make sure that tasks are given start times within their assigned time-window.

If there exist no feasible schedule to the subproblem in iteration $k$, a feasibility cut

$$
\sum_{i \in \overline{\mathcal{I}}^{k}}\left(1-x_{i \bar{q}_{i}^{k}}\right) \geq 1
$$

is added to the master problem. The feedback component of iteration $k$ is formulated as

$$
\left[\text { Feedback-LD }{ }^{k}\right] \sum_{i \in \overline{\mathcal{I}}^{k^{\prime}}}\left(1-x_{i \bar{q}_{i}^{k^{\prime}}}\right) \geq 1, \quad k^{\prime}=1, \ldots, k-1 .
$$


The application of LBBD in this section is straight-forward, but the best computational performance for both the LBBD scheme for single machine scheduling with multiple time-windows and sequence-dependent setup times in Paper D and the LBBD scheme for the avionics scheduling problem in Paper C, is obtained if feasibility cuts are strengthened. 



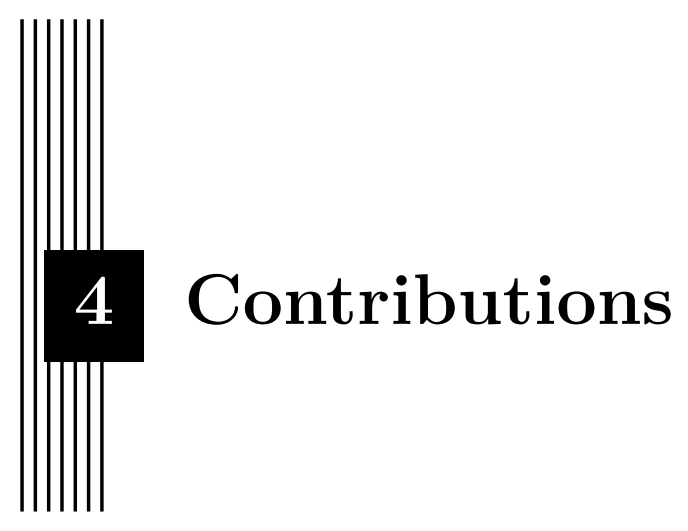

This chapter summarises the main contributions of Papers A-D in this thesis.

\subsection{An avionics scheduling design case}

The avionics scheduling design case was proposed by Saab Aeronautics, introduced in Paper A, and is of interest in the development of future avionic systems. The design case has particular properties due to the way communication works in the underlying avionic system. The design gives flexibility with respect to resource usage, but it increases the difficulty of the scheduling problem that needs to be solved. Compared to other work on avionics scheduling, the instances studied contain a larger number of tasks in general, and particularly, a larger number of tasks must be scheduled to perform a single end-to-end transfer of data in the system.

The avionics scheduling problem involves simultaneous scheduling of tasks and messages in a single problem. Constructing computationally efficient MIP and CP models for various aspects of this problem have been important parts of the work in Papers A-C. Without computationally efficient modelling, the decomposition approaches in Papers A-B and Paper C, and the matheuristic in Paper B, that all rely on solving a sequence of MIP and/or CP models, would not have been as successful. 


\subsection{Industrially relevant avionics scheduling instances}

The instances solved in this thesis have a number of tasks that far exceeds what has been reported in previous work on avionics scheduling. This provides challenges with respect to both developing methods and evaluating their performance. These challenges are increased by the lack of publicly available scheduling instances to evaluate methods on. For these reasons, we developed, in dialog with engineers and technical personnel from Saab, industrially relevant public large-scale avionics scheduling instances. These were presented in Paper B and are available at [2]. These instances have been used for computational evaluation of methods in Paper B and Paper C.

\subsection{Decomposition to exploit problem structure}

Two types of decomposition schemes are introduced in this thesis to address the computational challenges of the avionics scheduling problem. Both schemes rely on solving a master problem to find a preliminary schedule. The schedule is then used to construct a subproblem, which is a restriction of the original problem. If no feasible schedule is found by solving the subproblem, constraints are generated, and the master problem is resolved. However, the decomposition schemes differ in their master problems, the restrictions made, how the subproblem is solved, and how the constraints are generated.

In Paper A and Paper B, constraint generation procedures that solves MIP models were introduced and extended, respectively. In the master problem of this procedure, scheduling constraints are removed, but each task is assigned to an interval of time. The duration of this interval is slightly different in Paper A and Paper B. A subproblem is formed by forcing each task to be performed within the interval it was assigned to in the master problem solution. To the subproblem, soft scheduling constraints and an objective function to minimise the number of unfulfilled scheduling constraints are added. If no feasible schedule can be found within a time limit by solving the subproblem, a set of the unfulfilled scheduling constraints are added as hard constraints to both the master problem and the subproblem. This constraint generation procedure is able to solve instances with up to 37,000 tasks and 1700 messages but requires large quantities of RAM.

Paper C introduces a hybrid LBBD scheme that solves MIP and CP models. In the master problem of this scheme, which is solved by a MIP solver, scheduling and interval constraints are removed, but each message is assigned to a slot. A subproblem is formed by forcing each message to be performed in the slot it was assigned to in the master problem solution. To the subproblem, scheduling constraints are added and the constraints connecting message and task scheduling are propagated. This creates a task-scheduling-centred problem that can be efficiently solved with a CP solver. If the subproblem is infeasible, a feasibility cut is added to the master problem. To enhance the 
computational performance of the LBBD scheme, we propose an acceleration technique that solves additional CP models to strengthen feasibility cuts and to possibly find feasible solutions. This LBBD scheme is able to solve instances with up to 55,000 tasks and 2500 messages while using less memory than the methods in Paper A and Paper B.

\subsection{A matheuristic improvement}

In Paper B, a matheuristic is introduced to solve the master problem of the constraint generation procedure in Paper A. This matheuristic is a MIP-based ALNS. In each iteration of the ALNS, a schedule is found by solving a MIP model where certain constraints are treated as soft, and the model's objective function is to minimise the violations. In each iteration, a restriction is added to the MIP model by forcing a subset of tasks to be scheduled in restricted intervals based on the current schedule, while allowing the remaining tasks to change intervals to minimise the violations of the current schedule. When no violation remains, the soft constraints are reformulated as hard, the master problem objective function is added, and the ALNS is continued until no improvement has been found in the objective function for a number of iterations. This method solves instances with up to 55,000 tasks and 2500 messages.

\subsection{Accelerating logic-based Benders decomposition}

Besides a clever decomposition, acceleration techniques are key components when developing successful LBBD methods. Paper C and Paper D contribute to this research area by introducing and investigating acceleration techniques that rely on solving additional subproblems. The acceleration techniques are applied to LBBD schemes with MIP master problems and CP subproblems.

In Paper D, the computational performance of three known approaches to strengthen feasibility cuts in LBBD schemes are compared. To evaluate their performance, five instance sets for three problems with known decompositions are used. The decompositions share a common structure, where each master problem variable assigned to the value one implies that certain constraints in the subproblem must be active. The results shows that the DFBS cutstrengthening approach, that guarantee irreducible cuts, performs best.

In Paper $\mathrm{C}$, an acceleration technique that extends cut strengthening is introduced. By taking advantage of the information obtained from solving subproblems in the search of an irreducible cut, the acceleration technique makes educated guesses about which variables to fix to obtain a feasible solution. By constructing and solving restrictions of the original problem where such variables are fixed, feasible solutions are searched for. The acceleration technique is shown to be important for the computational performance of a LBBD scheme for the avionics scheduling problem. 



\section{Bibliography}

[1] J.M. van den Akker, C.P.M. van Hoesel, and M.W.P. Savelsbergh. "A polyhedral approach to single machine scheduling problems". In: Mathematical Programming 85.3 (1999), pp. 541-572. URL: https://doi. org/10.1007/s10107990047a.

[2] Avionics scheduling instances. https : / /gitlab.liu.se/eliro15/ avionics_inst/tree/master. Accessed: 2021-04-22.

[3] K.R. Baker and B. Keller. "Solving the single-machine sequencing problem using integer programming". In: Computers 8 Industrial Engineering 59.4 (2010), pp. 730-735. DOI: 10.1016/j.cie.2010.07.028.

[4] E. Balas. "On the facial structure of scheduling polyhedra". In: Mathematical Programming Study 24 (1985), pp. 179-218.

[5] P. Baptiste and R. Sadykov. "On scheduling a single machine to minimize a piecewise linear objective function: A compact MIP formulation". In: Naval Research Logistics 56.6 (2009), pp. 487-502. DOI: $10.1002 /$ nav. 20352.

[7] N. Boland, R. Clement, and H. Waterer. "A bucket indexed formulation for nonpreemptive single machine scheduling problems". In: Journal on Computing 28.1 (2016), pp. 14-30. DOI: 10.1287/ijoc.2015.0661.

[8] E.H. Bowman. "The schedule-sequencing problem". In: Operations Research 7.5 (1959), pp. 621-624. DOI: 10.1287/opre.7.5.621.

[9] E. Coban and J.N. Hooker. "Single-facility scheduling by logic-based Benders decomposition". In: Annals of Operations Research 210.1 (2013), pp. 245-272. DOI: 10.1007/s10479-011-1031-z. 
[10] F. Della Croce, F. Salassa, and V. T'Kindt. "A hybrid heuristic approach for single machine scheduling with release times". In: Computers 8 Operations Research 45.1 (2014), pp. 7-11. DOI: 10.1016/j.cor . 2013.11.016.

[11] M.E. Dyer and L.A. Wolsey. "Formulating the single machine sequencing problem with release dates as a mixed integer program". In: Discrete Applied Mathematics 26.2-3 (1990), pp. 255-270. DOI: 10.1016/0166218X (90) 90104-K.

[12] European Organisation for Civil Aviation Equipment (EUROCAE). "Software considerations in airborne systems and equipment certification, EUROCAE ED-12C". 2012.

[13] European Organisation for Civil Aviation Equipment (EUROCAE). "Design assurance guidance for airborne electronic hardware, EUROCAE ED-80". 2000.

[14] European Organisation for Civil Aviation Equipment (EUROCAE). "Integrated modular avionics (IMA) development guidance and certification considerations, EUROCAE ED-124". 2005.

[15] R. Fuchsen. "IMA NextGen: A new technology for the SCARLETT program". In: IEEE Aerospace and Electronic Systems Magazine 25.10 (2010), pp. 10-16. DOI: 10.1109/MAES.2010.5631720.

[16] M.R. Garey and D.S. Johnson. "Two-processor scheduling with starttimes and deadlines". In: SIAM Journal on Computing 6.3 (1977), pp. 416-426. DOI: $10.1137 / 0206029$.

[17] R.E. Gomory. "Outline of an algorithm for integer solution to linear programs". In: Bulletin of American Mathematical Society 64.5 (1958), pp. 275-278. DOI: 10.1090/S0002-9904-1958-10224-4.

[18] R.L. Graham, E.L. Lawler, J.K. Lenstra, and A.H.G. Rinnooy Kan. "Optimization and approximation in deterministic sequencing and scheduling: A survey". In: Annals of Discrete Mathematics 5.C (1979), pp. 287-326. DOI: 10.1016/S0167-5060(08)70356-X.

[19] M. Horn, G. R. Raidl, and E. Rönnberg. "A* search for prize-collecting job sequencing with one common and multiple secondary resources". In: Annals of Operations Research (2020). DOI: 10 .1007/s10479-02003550-7.

[20] W. A. Horn. "Some simple scheduling algorithms". In: Naval Research Logisitcs Quarterly 21.1 (1974), pp. 177-185. DOI: 10 . 1002 / nav . 3800210113.

[21] J. R. Jackson. Scheduling a production line to minimize maximum tardiness. Tech. rep. 1955, pp. 1-72.

[22] E. Karlsson. "Optimisation-based scheduling of an avionic system". Licentiate thesis. Linköping University, 2019. 
[23] E. Karlsson and E. Rönnberg. "Explicit modelling of multiple intervals in a constraint generation procedure for multiprocessor scheduling". In: Operations Research Proceedings 2017. Ed. by N. Kliewer, J.F. Ehmke, and R. Borndörfer. Springer International Publishing, 2018, pp. 567572. DOI: $10.1007 / 978-3-319-89920-6 \_75$.

[27] A.B. Keha, K. Khowala, and J.W. Fowler. "Mixed integer programming formulations for single machine scheduling problems". In: Computers 8 Industrial Engineering 56.1 (2009), pp. 357-367. DOI: 10.1016/j.cie. 2008.06 .008$.

[28] A. Kooli and M. Serairi. "A mixed integer programming approach for the single machine problem with unequal release dates". In: Computers E Operations Research 51.1 (2014), pp. 323-330. DOI: 10.1016/j.cor. 2014.06 .013$.

[29] J.B. Lasserre and M. Queyranne. "Generic scheduling polyhedra and a new mixed-integer formulation for single-machine scheduling". In: Proceedings of the 2nd International IPCO Conference. Ed. by E. Balas, G. Cornuéjols, and R. Kannan. 1992, pp. 136-149.

[30] J.Y-T. Leung. Handbook of scheduling: Algorithms, models, and performance Analysis. Boca Raton: Chapman \& Hall/CRC, 2004.

[31] A.S. Manne. "On the job-shop scheduling problem". In: Operations Research 8.2 (1960), pp. 219-223. DOI: 10.1287/opre.8.2.219.

[32] C.E. Miller, A.W. Tucker, and R.A. Zemlin. "Integer programming formulation of traveling salesman problems". In: Journal of the ACM (JACM) 7.4 (1960), pp. 326-329. DOI: 10.1145/321043.321046.

[33] G.L. Nemhauser and M.W.P. Savelsbergh. "A cutting plane algorithm for the single machine scheduling problem with release times". In: Combinatorial Optimization, NATO ASI Series F: Computer and System Sciences. Ed. by M. Akgül, H. Hamacher, and S. Tüfekçi. Vol. 82. 1992, pp. 63-83.

[34] A.A.B. Pritsker, L.J. Waiters, and P.M. Wolfe. "Multiproject scheduling with limited resources: A zero-one programming approach". In: Management Science 16.1 (1969), pp. 93-108. DOI: 10.1287/mnsc.16.1.93.

[35] M. Queyranne. "Structure of a simple scheduling polyhedron". In: Mathematical Programming 58.1-3 (1993), pp. 263-285. DOI: 10.1007 / BF01581271.

[36] Radio Technical Commission for Aeronautics (RTCA). "Software considerations in airborne systems and equipment certification, RTCA DO178 C". 2012.

[37] Radio Technical Commission for Aeronautics (RTCA). "Design assurance guidance for airborne electronic hardware, RTCA DO-254". 2000. 
[38] Radio Technical Commission for Aeronautics (RTCA). "Integrated modular avionics (IMA) development guidance and certification considerations, RTCA DO-297". 2005.

[39] M. Riedler, T. Jatschka, J. Maschler, and G.R. Raidl. "An iterative time-bucket refinement algorithm for a high-resolution resourceconstrained project scheduling problem". In: International Transactions in Operational Research (2017), pp. 1-41. DOI: 10.1111/itor.12445.

[40] E. Rönnberg. "Co-allocation of communication messages in an integrated modular avionic system". In: Operations Research Proceedings 2017. Ed. by N. Kliewer, J.F. Ehmke, and R. Borndörfer. Springer International Publishing, 2018, pp. 459-465. DOI: 10.1007/978-3-31989920-6_61.

[41] F. Rossi, P. van Beek, and T. Walsh. Handbook of constraint programming. Elsevier Science, 2006.

[42] B. B. Simmons. "A fast algorithm for single processor scheduling". In: Proceedings of the 19th Annual Symposium on Foundations of Computer Science. 1978, pp. 246-252.

[43] J.P. Sousa and L.A. Wolsey. "A time indexed formulation of nonpreemptive single machine scheduling problems". In: Mathematical Programming 54.1-3 (1992), pp. 353-367. DOI: 10.1007/BF01586059.

[44] H.M. Wagner. "An integer linear-programming model for machine scheduling". In: Naval research logistics quaterly 6.2 (1959), pp. 131140. DOI: $10.1002 /$ nav. 3800060205.

[45] H. Wang and W. Niu. "A review of key technologies of the distributed integrated modular avionics system". In: International Journal of Wireless Information Networks 25 (2018), pp. 358-369. DOI: 10.1007/s10776018-0412-5. 


\section{Papers}

The papers associated with this thesis have been removed for copyright reasons. For more details about these see:

http://urn.kb.se/resolve?urn=urn:nbn:se:liu:diva-173564 


\section{FACULTY OF SCIENCE AND ENGINEERING}

Linköping Studies in Science and Technology, Dissertation No. 2136, 2021 Department of Mathematics

Linköping University

SE-58183 Linköping, Sweden

www.liu.se 